University of Warwick institutional repository: http://go.warwick.ac.uk/wrap This paper is made available online in accordance with publisher policies. Please scroll down to view the document itself. Please refer to the repository record for this item and our policy information available from the repository home page for further information.

To see the final version of this paper please visit the publisher's website. Access to the published version may require a subscription.

Author(s): A. A. PINTO and D. A. RAND

Article Title: Teichmüller spaces and HR structures for hyperbolic surface dynamics

Year of publication: 2002

Link to published version:

http://dx.doi.org/10.1017/S0143385702000792

Publisher statement: None 


\title{
Teichmüller spaces and HR structures for hyperbolic surface dynamics
}

\author{
A. A. PINTO $\dagger$ and D. A. RAND \\ $\dagger$ DMA, Faculdade de Ciências, Universidade do Porto, 4000 Porto, Portugal \\ (e-mail: aapinto@fc.up.pt) \\ $\$$ Mathematics Institute, University of Warwick, Coventry CV4 7AL, UK \\ (e-mail:dar@maths.warwick.ac.uk)
}

(Received 23 June 2000 and accepted in revised form 9 January 2002)

\begin{abstract}
We construct a Teichmüller space for the $C^{1+}$-conjugacy classes of hyperbolic dynamical systems on surfaces. After introducing the notion of an HR structure which associates an affine structure with each of the stable and unstable laminations, we show that there is a one-to-one correspondence between these HR structures and the $C^{1+}$-conjugacy classes. As part of the proof we construct a canonical representative dynamical system for each HR structure. This has the smoothest holonomies of any representative of the corresponding $\mathrm{C}^{1+}$-conjugacy class. Finally, we introduce solenoid functions and show that they provide a good Teichmüller space.
\end{abstract}

\section{Introduction}

In this paper we study the flexibility of smooth hyperbolic dynamics on surfaces. By the flexibility of a given topological model of hyperbolic dynamics we mean the extent of different smooth realizations of this model. Thus a typical result provides a Teichmüller space or a moduli space to parametrize these realizations. In this paper we will construct Teichmüller spaces for hyperbolic sets of surface diffeomorphisms with one-dimensional stable and unstable manifolds including Anosov diffeomorphisms, attractors and Smale horseshoes. In a later paper we extend these results to pseudo-Anosov systems.

To be effective it is important that these Teichmüller spaces should be easily characterized. For example, for Anosov diffeomorphisms of the torus that are either $C^{\infty}$ or $C^{2}$ and preserve a smooth invariant measure, the eigenvalue spectrum is known to be a complete invariant of smooth conjugacy $[4,5]$. However, it is not clear which eigenvalue spectra are realized by such systems. Thus these do not make up a good Teichmüller space. The Teichmüller spaces that we construct do not suffer from this and they usually consist of easily characterized Hölder functions. Moreover, for hyperbolic systems on surfaces other than Anosov systems not only are the eigenvalue spectra difficult to characterize, they are also only a complete invariant of Lipschitz conjugacy. 
A related Teichmüller space for Anosov diffeomorphisms of tori has been constructed by Cawley [2]. This is in terms of cohomology classes of Hölder cocycles defined on the torus. Its effectiveness for Anosov systems relies on the fact that the Lipschitz and $\mathrm{C}^{1+}$ theories coincide. This is not the case for other hyperbolic systems and so Cawley's Teichmüller description in terms of Hölder cocycles will not work in the general case that we treat here.

1.1. Teichmüller spaces. We now give a more precise description of our results. Consider a $C^{1+}$ diffeomorphism $\dagger f$ of a compact surface $M$ which has a hyperbolic invariant subset $\Lambda$. We assume throughout that $f \mid \Lambda$ is topologically transitive and that $\Lambda$ has a local product structure [17]. To avoid having to repeat this fact we adopt the convention that by a hyperbolic set we mean one with these extra properties. We allow both the case where $\Lambda=M$ (so that $f$ is Anosov and $M \cong \mathbb{T}^{2}[3,9]$ ) and the case where $\Lambda$ is a proper subset (e.g. a horseshoe or an attractor with one-dimensional unstable manifolds such as the Plykin attractor).

We start by introducing the notion of a $H R$ (Hölder ratio) structure. We consider affine structures on the stable and unstable laminations in $\Lambda$ (\$3). These are defined in terms of a pair of ratio functions $r^{s}$ and $r^{u}$. If $r^{s}$ and $r^{u}$ are Hölder continuous and invariant under $f$ then we call the associated structure an HR structure. Theorem 5.1 gives a oneto-one correspondence between HR structures and the $C^{1+}$ conjugacy classes of $f \mid \Lambda$. The main step in the proof of this and related results is to show that, given an HR structure, there is a canonical construction of a representative in the corresponding conjugacy class. By Theorem 5.3, this representative has the following maximum smoothness property: the holonomy maps for the representative are as smooth as those of any diffeomorphism that is $C^{1+}$ conjugate to it. In particular, if there is an affine diffeomorphism with this HR structure, then this representative is the affine diffeomorphism.

It is interesting to note that when we consider the $C^{1+}$ realizations of a particular topological model then the stable and unstable ratio functions are independent in the following sense. If $r^{s}$ is a stable ratio function for some realization and $r^{u}$ is the unstable ratio function for some other realization then there is a realization that has the pair $\left(r^{s}, r^{u}\right)$ as its HR structure.

In $\S 6$ we define solenoid functions. Each HR structure has a pair $\left(s^{s}, s^{u}\right)$ of these corresponding to the stable and unstable laminations of $\Lambda$ associated with it. The solenoid functions $s^{s}$ and $s^{u}$ are the restrictions of the ratio functions $r^{s}$ and $r^{u}$, respectively, to a set determined by a Markov partition of $f$. Theorem 6.1 states that there is a one-to-one correspondence between Hölder solenoid function pairs and HR structures. Since these solenoid function pairs form a nice space with a simply characterized completion they provide a good Teichmüller space. For example, in the classical case of Smale horseshoes the Teichmüller space is the set of all pairs of positive Hölder continuous functions with the domain $\{0,1\}^{\mathbb{N}}$.

THEOREM 1.1. (Teichmüller space) The natural map $c$ which associates a solenoid function pair to a $\mathrm{C}^{1+}$ conjugacy class is injective and has a well-characterized image and a natural inverse on it.

$\dagger$ See the note in $\$ 1.2$ regarding our meaning of $C^{1+}$. 
Although we will not prove it in this paper we mention here that the completion of the image of $c$ is the set of pairs of continuous solenoid functions which is a closed subset of a Banach space. They correspond to $f$-invariant affine structures on the stable and unstable laminations for which the holonomies are uniformly asymptotically affine as defined in [19].

This Teichmüller space is also important because it enables us to construct interesting classes of smooth hyperbolic systems. For example, in [13] we use it to construct all such systems with an invariant measure with a given geometric measure class (such as all Anosov diffeomorphisms with an invariant measure that is absolutely continuous with respect to a two-dimensional Lebesgue measure). Another interesting application of this Teichmüller space appears in [15] which studies rigidity for diffeomorphisms on surfaces. For example, a $C^{r}$ Anosov map with $C^{2+\varepsilon}$ holonomies is $C^{r-\alpha}$ conjugated to a hyperbolic toral automorphism for every small $\alpha>0$, where $0<\varepsilon<1$.

This paper extends the work of Sullivan and of the authors from one-dimensional expanding dynamics (see [10], [16] and [18]) to the context of hyperbolic dynamics on surfaces.

\subsection{Conventions.}

(1) Smoothness. In this paper, when we say that a map, atlas or structure is $C^{r}$ we include the case $C^{k+}$ where $k$ is a positive integer. For maps $f$ this means that $f$ is $C^{k+\alpha}$ for some $0<\alpha \leq 1$, i.e. $C^{k}$ with $\alpha$-Hölder continuous $k$ th-order derivatives. For an atlas or structure this means that each pair of charts in the atlas or structure are $C^{k+\alpha}$ compatible for some $0<\alpha \leq 1$ where the $\alpha$ might depend upon the charts. In the case of an atlas, we suppose that (i) one can choose $\alpha$ to be independent of the charts and (ii) the overlap maps have $C^{k+\alpha}$ norm bounded independent of the charts considered. This is immediately verified if the number of charts contained in the $C^{k+}$ atlas is finite. Thus a $C^{k+}$ atlas is $C^{k+\alpha}$, for some $0<\alpha \leq 1$. This is not the case for $C^{k+}$ structures.

(2) Stable and unstable superscripts. Throughout the paper we will use the following notation: we use $\iota$ to denote an element of the set $\{s, u\}$ of the stable and unstable superscripts and $\iota^{\prime}$ to denote the element of $\{s, u\}$ that is not $\iota$. In the main discussion we will often refer to objects which are qualified by $\iota$ such as, for example, an $\iota$-leaf. This means a leaf which is a leaf of the stable lamination if $\iota=s$ or the unstable lamination if $\iota=u$. In general the meaning should be quite clear.

(3) $f_{\iota}$. We define the map $f_{\iota}=f$ if $\iota=u$ or $f_{\iota}=f^{-1}$ if $\iota=s$.

(4) $\mathcal{O}$ notation. We use the notation $\phi=\mathcal{O}(\psi(x))$ to indicate that for all $x,|\phi(x)|<$ $c|\psi(x)|$ where $c>0$ is a constant which depends only upon quantities that are explicitly mentioned. Thus $\phi(n)=\mathcal{O}\left(v^{n}\right)$ means that $|\phi(n)|<c v^{n}$ for some constant $c$ as above. Similarly we use $\phi=\mathcal{O}(\psi(x))$ to indicate that, for all $x$, $c_{1}|\psi(x)|<|\phi(x)|<c_{2}|\psi(x)|$ where $c_{1}$ and $c_{2}$ are constants which depend only upon quantities that are explicitly mentioned. 
(5) Interval notation. We also use the notation of interval arithmetic for some inequalities where

- if $I$ and $J$ are intervals then $I+J, I . J$ and $I / J$ have the obvious meaning as intervals;

- if $I=\{x\}$ then we often denote $I$ by $x$; and

- $I \pm \varepsilon$ denotes the interval consisting of those $x$ such that $|x-y|<\varepsilon$ for all $y \in I$.

Thus $\phi(n) \in 1 \pm \mathcal{O}\left(v^{n}\right)$ means that there exists a constant $c>0$ depending only upon explicitly mentioned quantities such that for all $n \geq 0,1-c v^{n}<\phi(n)<1+c v^{n}$.

\section{The topological starting point}

The basic topological object of our study will be a triple $(f, \Lambda, \mathcal{W})$ consisting of an invariant set $\Lambda$, a homeomorphism $f: \Lambda \rightarrow \Lambda$ and a topological lamination structure $\mathcal{W}$. To consider the meaning of this we consider how such objects arise in hyperbolic dynamics.

Let $\phi$ be a $C^{1+\gamma}$ diffeomorphism of the surface $M$ and suppose that $\phi$ has a topologically transitive hyperbolic invariant set $\Lambda$ with a local product structure [17]. If $f=\phi \mid \Lambda$, let $f_{s}=f^{-1}, \phi_{s}=\phi^{-1}, f_{u}=f$ and $\phi_{u}=\phi$. Fix a metric $d$ on $M$. For $\iota=s$ or $u$, if $x \in \Lambda$ we denote the local stable and unstable manifolds through $x$ by

$$
W^{l}(x, \varepsilon)=\left\{y \in M: d\left(\phi_{l}^{-n}(x), \phi_{l}^{-n}(y)\right) \leq \varepsilon, \text { for all } n \geq 0\right\} .
$$

These sets are respectively contained in the stable and unstable manifolds

$$
W^{l}(x)=\bigcup_{n \geq 0} f_{l}^{n}\left(W^{l}\left(f_{l}^{-n}(x), \varepsilon_{0}\right)\right)
$$

which are the image of a $C^{1+\gamma}$ immersion $\lambda_{x}: \mathbb{R} \rightarrow M$.

Definition 2.1. A full l-leaf segment $I$ is a subset of $W^{\imath}(x)$ of the form $\lambda_{x}\left(I_{1}\right)$ where $I_{1}$ is an open subinterval in $\mathbb{R}$. A $\iota$-leaf segment is the intersection with $\Lambda$ of a full $\iota$-leaf segment. The collection of all subsets of $\iota$-leaf segments is denoted by $\mathcal{D}^{\iota}$.

The endpoints of such a full $\iota$-leaf segment are the points $\lambda_{x}(u)$ and $\lambda_{x}(v)$ where $u$ and $v$ are the endpoints of $I_{1}$. The endpoints of such a $\iota$-leaf segment $I$ are the points of the minimal full $\iota$-leaf segment containing $I$.

We say that two embeddings $i: I \rightarrow \mathbb{R}$ and $j: J \rightarrow \mathbb{R}$ where $I, J \in \mathcal{D}^{l}$ are compatible if whenever $I \cap J \neq \emptyset$ then $j \circ i^{-1}: i(I \cap J) \rightarrow j(I \cap J)$ extends to a homeomorphism of the real line. A topological l-lamination atlas is a set of such maps whose domains cover $\Lambda$ and which are pairwise compatible with each other. A topological ı-lamination structure is the maximal set of such embeddings compatible with such an atlas. A topological lamination structure $\mathcal{W}=\left(\mathcal{W}^{s}, \mathcal{W}^{u}\right)$ is a pair made up from a topological $s$-lamination structure $\mathcal{W}^{s}$ and a topological $u$-lamination structure $\mathcal{W}^{u}$.

By the Stable Manifold Theorem, the diffeomorphism $\phi$ defines such a lamination structure $\mathcal{W}_{\phi}$ as follows. For each $\iota$-leaf segment $I$ consider the mappings $i: I \rightarrow \mathbb{R}$ which are the restrictions to $I$ of topological charts of the submanifold structure of $I$. These are 
all compatible with each other and define a topological $\iota$-lamination atlas. The topological lamination structure $\mathcal{W}_{\phi}$ is defined by these two atlases.

More unusually we also desire to highlight the $C^{1+}$ structure on $M$ in which $\phi$ is a diffeomorphism. By a $C^{1+}$ structure on $M$ we mean a maximal set of charts with open domains in $M$ such that the union of their domains cover $M$ and whenever $U$ is an open subset contained in the domains of any two of these charts $i$ and $j$ then the overlap map $j \circ i^{-1}: i(U) \rightarrow j(U)$ is $C^{1+\alpha}$, where $\alpha>0$ depends on $i, j$ and $U$. We note that by the compactness of $M$, given such a $C^{1+}$ structure on $M$, there is an atlas consisting of a finite set of these charts which cover $M$ and for which the overlap maps are $C^{1+\alpha}$ compatible and uniformly bounded in the $C^{1+\alpha}$ norm, where $\alpha>0$ just depends upon the atlas. We denote by $\mathcal{S}_{\phi}$ the $C^{1+}$ structure on $M$ in which $\phi$ is a diffeomorphism. Usually one is not concerned with this as, given two such structures, there is a homeomorphism of $M$ sending one onto the other and thus, from this point of view, all such structures can be identified. For our discussion it will be important to maintain the identity of the different smooth structures on $M$.

Our approach in this paper is to fix the triple $(f, \Lambda, \mathcal{W})$ and to consider its $C^{1+}$ hyperbolic realizations which are defined as follows. Consider a $C^{1+}$ diffeomorphism $\phi$ of a surface with an associated $C^{1+}$ structure $\mathcal{S}_{\phi}$ and assume that $\phi$ possesses a hyperbolic invariant set $\Lambda_{\phi}$ with associated lamination structure $\mathcal{W}_{\phi}$. Then we say that $\phi$ is a $C^{1+}$ hyperbolic realization of $(f, \Lambda, \mathcal{W})$ if there is a topological conjugacy $h_{\phi}$ between $\phi \mid \Lambda_{\phi}$ and $f \mid \Lambda$ which sends the lamination structure $\mathcal{W}_{\phi}$ onto $\mathcal{W}$. We say that two of these $C^{1+}$ hyperbolic realizations $\phi$ and $\psi$ are $C^{1+}$ conjugate if $h_{\psi}^{-1} \circ h_{\phi}$ has a $C^{1+}$ diffeomorphic extension to an open set of $M$ containing $\Lambda_{\phi}$. By diffeomorphic we mean that it is a diffeomorphism between the structures $\mathcal{S}_{\phi}$ and $\mathcal{S}_{\psi}$. In this paper, we are essentially interested in studying and classifying the $C^{1+}$ conjugacy classes of the $C^{1+}$ hyperbolic realizations of $(f, \Lambda, \mathcal{W})$.

Suppose that $\phi$ is a $C^{1+}$ hyperbolic realization of $f$. If $\hat{h}_{\phi}$ is a homeomorphism which extends the topological conjugacy $h_{\phi}: \Lambda_{\phi} \rightarrow \Lambda$ to a neighbourhood of $\Lambda$ in $M$, then we obtain a $C^{1+}$ hyperbolic realization $\psi=\hat{h}_{\phi} \circ \phi \circ \hat{h}_{\phi}^{-1}$ of $f$ with the associated $C^{1+}$ structure $\mathcal{S}_{\psi}=\left(\hat{h}_{\phi}\right)_{*} \mathcal{S}_{\phi}$. Furthermore, $\psi$ is $C^{1+}$ conjugated to $\phi$ and $\Lambda_{\psi}=\Lambda$. Hence, to study the $C^{1+}$ conjugacy classes of hyperbolic realizations of $f$, we can just consider the $C^{1+}$ hyperbolic realizations $\phi$ with $\Lambda_{\phi}=\Lambda$ which we will do from now on for simplicity of our exposition.

2.1. Rectangles. Since there is a hyperbolic realization, for $0<\varepsilon<\varepsilon_{0}$ there is a $\delta=\delta(\varepsilon)>0$, such that for all points $z, w \in \Lambda$ with $d(w, z)<\delta, W^{s}(z, \varepsilon)$ and $W^{u}(w, \varepsilon)$ intersect in a unique point $[w, z]$. Here $d$ is any smooth metric on $M$. Since we assume that the hyperbolic set has a local product structure, we have that $[w, z] \in \Lambda$. Furthermore, the following properties are satisfied: (i) $[w, z]$ varies continuously with $w, z \in \Lambda$; (ii) the bracket map is continuous on a $\delta$-uniform neighbourhood of the diagonal in $\Lambda \times \Lambda$; and (iii) whenever both sides are defined $f([z, w])=[f(z), f(w)]$. Note that the bracket map does not really depend on $\delta$ provided $\delta$ is sufficiently small.

Let us emphasize that it is a standing hypothesis that all the hyperbolic sets considered here have such a local product structure. 
A rectangle $R$ is a subset of $\Lambda$ which is (i) closed under the bracket, i.e. $x, y \in R \Longrightarrow$ $[x, y] \in R$; and (ii) proper, i.e. is the closure of its interior in $\Lambda$. This definition imposes the condition that a rectangle has always to be proper which is more restrictive than the usual one which only insists on the closure condition.

If $\ell$ and $\ell^{\prime}$ are, respectively, stable and unstable leaf segments then we denote by $\left[\ell, \ell^{\prime}\right]$ the set consisting of all points of the form $\left[z, z^{\prime}\right]$ with $z \in \ell$ and $z^{\prime} \in \ell^{\prime}$. We note that if the stable and unstable leaf segments $\ell$ and $\ell^{\prime}$ are closed then the set $\left[\ell, \ell^{\prime}\right]$ is a rectangle. Conversely in this two-dimensional situation, any rectangle $R$ has a product structure in the following sense: for each $x \in R$ there are closed stable and unstable leaf segments of $\Lambda$, $\ell^{s}(x, R) \subset W^{s}(x)$ and $\ell^{u}(x, R) \subset W^{u}(x)$ such that $R=\left[\ell^{s}(x, R), \ell^{u}(x, R)\right]$. The leaf segments $\ell^{s}(x, R)$ and $\ell^{u}(x, R)$ are called stable and unstable spanning leaf segments for $R$. The interior of $R$ is given by int $R=\left[\operatorname{int} \ell^{s}(x, R)\right.$, int $\left.\ell^{u}(x, R)\right]$, and the boundary of $R$ is given by $\partial R=\left[\partial \ell^{s}(x, R), \ell^{u}(x, R)\right] \cup\left[\ell^{s}(x, R), \partial \ell^{u}(x, R)\right]$.

2.2. Markov partitions. A Markov partition of $f$ is a collection $\mathcal{R}=\left\{R_{1}, \ldots, R_{m}\right\}$ of rectangles such that (i) $\Lambda \subset \bigcup_{i=1}^{m} R_{i}$; (ii) $R_{i} \cap R_{j}=\partial R_{i} \cap \partial R_{j}$ for all $i$ and $j$; (iii) if $x \in$ int $R_{i}$ and $f x \in$ int $R_{j}$ then

(a) $f\left(\ell^{s}\left(x, R_{i}\right)\right) \subset \ell^{s}\left(f x, R_{j}\right)$ and $f^{-1}\left(\ell^{u}\left(f x, R_{j}\right)\right) \subset \ell^{u}\left(x, R_{i}\right)$; and

(b) $f\left(\ell^{u}\left(x, R_{i}\right)\right) \cap R_{j}=\ell^{u}\left(f x, R_{j}\right)$ and $f^{-1}\left(\ell^{s}\left(f x, R_{j}\right)\right) \cap R_{i}=\ell^{s}\left(x, R_{i}\right)$.

The last condition means that $f\left(R_{i}\right)$ goes across $R_{j}$ just once. In fact, it follows from condition (a) provided the rectangles $R_{j}$ are chosen to be sufficiently small [8]. The rectangles which make up the Markov partition are called Markov rectangles.

By the existence of a smooth realization our topological model $(f, \Lambda, \mathcal{W})$ has such a local product structure and a Markov partition [17]. Moreover, it is clear that they also only depend upon $(f, \Lambda, \mathcal{W})$ and are independent of the hyperbolic realization $\phi$ and the smooth structure $\mathcal{S}_{\phi}$ on $M$.

For $\iota=s$ or $u$ a $\iota$-leaf primary cylinder is a spanning $\iota$-leaf segment of a Markov rectangle. A $\iota$-leaf $n$-cylinder is an $\iota$-leaf segment $I$ such that $f_{l}^{n} I$ is an $\iota$-leaf primary cylinder. For $n>1$, a $\iota$-leaf $n$-gap is a pair of distinct points $x, y$ such that

(i) for some rectangle $R$ containing $x$ and $y$ and an embedding $i: \ell^{\iota}(x, R) \rightarrow \mathbb{R}$ in the topological lamination structure, $\{x, y\}=i^{-1}(J)$ for some non-trivial closed interval $J$ in $\mathbb{R}$; and

(ii) for $j=0, \ldots, n-1, f^{j} x$ lies in a Markov rectangle $R_{i_{j}}$ and $f^{j} y \in \ell\left(f^{j} x, R_{i_{j}}\right)$ but this is not the case for $j=n$.

A primary $\iota$-leaf gap is the image under $f_{\iota}$ of an $\iota$-leaf 1-gap.

We say that a rectangle $R$ is an $\left(n_{s}, n_{u}\right)$-rectangle if there is an $x \in R$ such that, for $\iota=s$ and $u$, the spanning leaf segments $\ell^{\iota}(x, R)$ are either an $\iota$-leaf $n_{\iota}$-cylinder or the union of two such cylinders with a common endpoint. The reason for allowing the possibility of the spanning leaf segments being inside two touching cylinders is to allow us to regard geometrically very small rectangles intersecting a common boundary of two Markov rectangles to be small in the sense of having $n_{s}$ and $n_{u}$ large. 


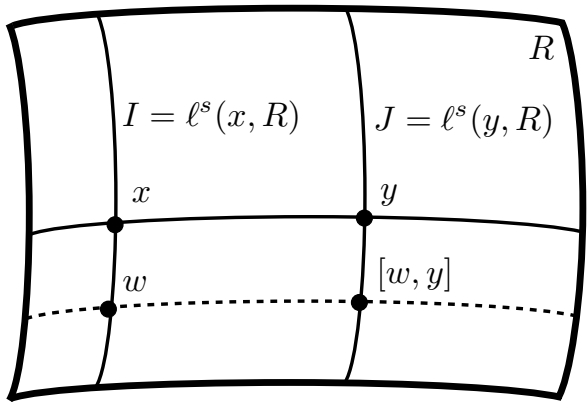

FIGURE 1. A basic stable holonomy.

Definition 2.2. If $x, y \in \Lambda$ and $x \neq y$ then define $d_{\Lambda}(x, y)=2^{-n}$ where $n$ is the greatest integer such that both $x$ and $y$ are contained in an $\left(n_{s}, n_{u}\right)$-rectangle with $n_{s}, n_{u} \leq n$. Similarly if $I$ and $J$ are $\iota$-leaf segments then $d_{\Lambda}(I, J)=2^{-n}$ where $n_{s}$ and $n_{u}$ are the greatest integers such that both $I$ and $J$ are contained in an $\left(n_{s}, n_{u}\right)$-rectangle and $n=n_{\iota^{\prime}}$. Here $\iota^{\prime}$ is the element of $\{s, u\}$ that is not $\iota$.

2.3. Basic holonomies. We concentrate on the stable holonomies. The unstable holonomies are entirely analogous. Suppose that $x$ and $y$ are two points inside any rectangle $R$ of $\Lambda$ such that $y \in \ell^{u}(x, R)$. Let $I$ and $J$ be two stable leaf segments respectively containing $x$ and $y$ and inside $R$. Then we define $\theta: I \rightarrow J$ by $\theta(w)=[w, y]$. Such maps are called the basic stable holonomies (in the rectangle $R$ ) (see Figure 1). They generate the pseudo-group of all stable holonomies. Similarly we define the unstable basic holonomies.

2.4. Foliated lamination atlas. In this section when we refer to a $C^{r}$ object $r$ is allowed to take the values $k+\alpha$ where $k$ is a positive integer and $0<\alpha \leq 1$.

Two charts $i$ and $j$ in $\mathcal{W}^{\iota}$ are $C^{r}$ compatible if whenever $U$ is an open subset of an $\iota$-leaf segment contained in the domains of $i$ and $j$ then $j \circ i^{-1}: i(U) \rightarrow j(U)$ extends to a $C^{r}$ diffeomorphism of the real line. Such maps are called chart overlap maps. A bounded $C^{r}$ ı-lamination atlas $\mathcal{A}^{\iota}$ is a set of such charts which (a) cover $\Lambda$; (b) are pairwise $C^{r}$ compatible; and (c) have chart overlap maps which are uniformly bounded in the $C^{r}$ norm.

Let $\mathcal{A}^{\iota}$ be a bounded $C^{1+\alpha}$ l-lamination atlas, with $0<\alpha \leq 1$. If $i: I \rightarrow \mathbb{R}$ is a chart of $\mathcal{A}^{\iota}$ defined on the leaf segment $I$ and $K$ is a leaf segment in $I$ then we define $|K|_{i}$ to be the length of the minimal closed interval containing $i(K)$. Since the atlas is bounded, if $j: J \rightarrow \mathbb{R}$ is another chart in $\mathcal{A}^{\iota}$ defined on the leaf segment $J$ which contains $K$ then the ratio between the lengths $|K|_{i}$ and $|K|_{j}$ is universally bounded away from 0 and $\infty$. If $K^{\prime} \subset I \cap J$ is another such segment then we can define the ratio $r_{i}\left(K: K^{\prime}\right)=|K|_{i} /\left|K^{\prime}\right|_{i}$. Although this ratio depends upon $i$, the ratio is exponentially determined in the sense that if $T$ is the smallest segment containing both $K$ and $K^{\prime}$ then

$$
r_{j}\left(K: K^{\prime}\right) \in\left(1 \pm \mathcal{O}\left(|T|_{i}^{\alpha}\right)\right) r_{i}\left(K: K^{\prime}\right) .
$$


This follows from using the Mean Value Theorem and the $C^{1+\alpha}$ smoothness of the overlap maps.

Definition 2.3. A $C^{r}$ lamination atlas has bounded geometry if (i) for all pairs $I_{1}, I_{2}$ of $\iota$ leaf $n$-cylinders or $\iota$-leaf $n$-gaps with a common point, we have that $\left|I_{1}\right|_{i} /\left|I_{2}\right|_{i}$ is uniformly bounded away from 0 and $\infty$ with the bounds being independent of $i, I_{1}, I_{2}$ and $n$; and (ii) for all endpoints $x$ and $y$ of an $\iota$-leaf $n$-cylinder or $\iota$-leaf $n$-gap $I$, we have that $|I|_{i} \leq \mathcal{O}\left(\left(d_{\Lambda}(x, y)\right)^{\beta}\right)$ and $d_{\Lambda}(x, y) \leq \mathcal{O}\left(|I|_{i}^{\beta}\right)$, for some $0<\beta<1$, independent of $i, I$ and $n$.

Definition 2.4. A bounded $C^{r}$ t-lamination is $C^{r}$-foliated if (i) the basic holonomies are $C^{r}$; and (ii) for every rectangle $R$ the $C^{r}$ norm of the holonomies in $R$ are uniformly in this atlas.

The following result relates smoothness of the holonomy with ratio distortion and will be used several times. It follows directly from Theorem 3 of [11].

Proposition 2.5. Suppose that $\theta: I \rightarrow J$ is a basic -holonomy for the rectangle $R$ and $i: I \rightarrow \mathbb{R}$ and $j: J \rightarrow \mathbb{R}$ are in $\mathcal{A}^{\iota}$. The holonomy $\theta: I \rightarrow J$ is $C^{1+\beta}$ for every $0<\beta<\alpha$ with respect to the charts of the lamination atlas $\mathcal{A}^{\iota}$ if and only if for every $0<\beta<\alpha$ and for all $I_{1}, I_{2} \subset I$ with $I_{1}$ a leaf $n$-cylinder and $I_{2}$ a leaf $n$-cylinder or $a$ leaf $n$-gap, we have

$$
\left|\log \frac{\left|j \theta I_{1}\right|}{\left|j \theta I_{2}\right|} \frac{\left|i I_{2}\right|}{\left|i I_{1}\right|}\right| \leq \mathcal{O}_{R}\left(|i K|^{\beta}\right)
$$

whenever $K$ is an t-leaf segment containing $I_{1}$ and $I_{2}$. Moreover, there are some $0<$ $\beta, \eta<\alpha$ and some affine map $a: \mathbb{R} \rightarrow \mathbb{R}$ such that

$$
\left\|j \circ \theta \circ i^{-1}-a\right\|_{C^{1+\eta}} \leq \mathcal{O}_{R}\left(\left(d_{\Lambda}(I, J)\right)^{\beta}\right)
$$

if and only if there are some $0<\beta, v<1$ such that for all $I_{1}$ and $I_{2}$ as before we have

$$
\left|\log \frac{\left|j \theta I_{1}\right|}{\left|j \theta I_{2}\right|} \frac{\left|i I_{2}\right|}{\left|i I_{1}\right|}\right| \leq \mathcal{O}_{R}\left(\left(d_{\Lambda}(I, J)\right)^{\beta} v^{n}\right) .
$$

If $L \subset \mathbb{R}$ then by $|L|$ we mean the Euclidean length of the minimal interval in $\mathbb{R}$ containing $L$.

2.5. $C^{1+}$ foliated atlas associated with $C^{1+}$ realizations. Let $\phi$ be a $C^{r}$ hyperbolic realization of $(f, \Lambda, \mathcal{W})$. By the Stable Manifold Theorem, the full $\iota$-leaf segments $I$ are $C^{r}$ submanifolds of $M$, which are the image of $C^{r}$ embeddings $\lambda: I_{1} \subset \mathbb{R} \rightarrow M$. Hence, the restrictions of $\lambda^{-1}$ to $\lambda(I \subset \Lambda)$ form a $C^{r}$ lamination atlas $\mathcal{A}_{\phi}^{\iota}$.

Let $\rho$ be a Riemannian metric which is $C^{1+\gamma}$, with $0<\gamma \leq 1$. This is a metric which in the charts of some atlas on $M$ is given by $g_{11} d x^{2}+g_{12} d x d y+g_{22} d y^{2}$ where the functions $g_{i j}$ are Holder continuous with exponent $\gamma$ and are uniformly bounded in the $C^{\gamma}$-norm. The $C^{r}$ embeddings $\lambda: I_{1} \subset \mathbb{R} \rightarrow M$ of the full $\iota$-leaf segments $I$ can be reparametrized such that they are isometries between the Euclidean metric on $I_{1}$ and the induced $\rho$ metric on $I$. By construction, these reparametrizations $\lambda: I_{1} \subset \mathbb{R} \rightarrow M$ are $C^{1+\gamma}$ embeddings. Hence, the restrictions of $\lambda^{-1}$ to $\lambda\left(I_{1} \cap \Lambda\right)$ form a $C^{1+\gamma}$ lamination 
atlas $\mathcal{A}^{\iota}(\rho)$. We note that the charts in $\mathcal{A}^{\iota}(\rho)$ are $C^{1+\gamma}$ compatible with the charts in $\mathcal{A}_{\phi}^{\iota}$. By Lemma 4.2 in [14], the lamination atlas $\mathcal{A}^{\iota}(\rho)$ has bounded geometry. By Theorem 2.2 in [14], the lamination atlas $\mathcal{A}^{\iota}(\rho)$ is $C^{1+\alpha}$-foliated, for some $0<\alpha \leq 1$, and the $\iota$-basic holonomies satisfy (2.2), with respect to the charts in this atlas.

\section{HR structures}

In this section we introduce HR structures. These associate an affine structure with each stable and unstable leaf segment in such a way that these vary Hölder continuously with the leaf. Our goal is to prove that a $C^{1+}$ conjugacy class is determined by its HR structure and vice versa.

An affine structure on a stable or unstable leaf in $\Lambda$ is equivalent to a ratio function $r(I: J)$ which can be thought of as prescribing the ratio of the size of two leaf segments $I$ and $J$ in the same stable or unstable leaf. A ratio function $r(I: J) \in(0, \infty)$ is defined for any pair of leaf segments $I$ and $J$ which are both contained in some larger segment. Although for notational clarity we write $r(I: J)$, in fact $r$ only depends upon the endpoints $x$ and $y$ of $I$ and $z$ and $w$ of $J$. Thus the domain of $r$ is the set of such quadruples $(x, y, z, w)$. Since these can be considered as forming a subset of $\Lambda^{4}$ they inherit its topology. We demand that $r$ is continuous in this topology. Moreover, it must satisfy

$$
r(I: J)=r(J: I)^{-1} \quad \text { and } \quad r\left(I_{1} \cup I_{2}: K\right)=r\left(I_{1}: K\right)+r\left(I_{2}: K\right)
$$

provided $I_{1}$ and $I_{2}$ intersect at most in one of their endpoints.

Definition 3.1. We say that $r$ is an $\iota$-ratio function if it satisfies these conditions and, moreover, (i) $r$ is invariant under $f$, i.e. $r(I: J)=r(f I: f J)$ for all $\iota$-leaf segments; and (ii) for every basic $\iota$-holonomy map $\theta: I \rightarrow J$ between the leaf segment $I$ and the leaf segment $J$ defined with respect to a rectangle $R$ and for every $\iota$-leaf segment $I_{1} \subset I$ and every $\iota$-leaf segment or gap $I_{2} \subset I$,

$$
\left|\log \frac{r\left(\theta I_{1}: \theta I_{2}\right)}{r\left(I_{1}: I_{2}\right)}\right| \leq \mathcal{O}\left(\left(d_{\Lambda}(I, J)\right)^{\varepsilon}\right)
$$

where $\varepsilon \in(0,1)$ depends upon $r$ and the constant of proportionality also depends upon $R$, but not on the segments considered.

Definition 3.2. An HR structure is a pair $\left(r^{s}, r^{u}\right)$ consisting of a stable and an unstable ratio function.

3.1. $C^{1+}$ foliated atlases associated with an HR structure. Given an $\iota$-ratio function $r$, we define the embeddings $e: I \rightarrow \mathbb{R}$ by

$$
e(x)=r(\ell(\xi, x), \ell(\xi, R))
$$

where $\xi$ is an endpoint of the $\iota$-leaf segment $I, R$ is a Markov rectangle containing $\xi$ (but not necessarily containing $I$ ) and $\ell(\xi, x)$ is the $\iota$-leaf segment with endpoints $x$ and $\xi$. For this definition it is not necessary that $R$ contains $I$. We denote the set of all these embeddings $e$ by $\mathcal{A}(r)$. 
The embeddings $e$ of $\mathcal{A}(r)$ have overlap maps with affine extensions, therefore the atlas $\mathcal{A}(r)$ extends to a $C^{1+\alpha}$ lamination structure, $\mathcal{L}(r)$. In Proposition 3.3, we prove that the atlas $\mathcal{A}(r)$ has a bounded geometry, and in Proposition 3.5 we prove that in this the basic holonomies are $C^{1+\beta}$ for some $0<\beta<1$. Thus this lamination structure is $C^{1+}$ foliated in the sense of Definition 2.4. Moreover, it is a unique structure compatible with $r$ in the sense that it and $r$ induce the same $C^{1+}$ structures on leaf segments.

Proposition 3.3. Let $r$ be an l-ratio function. Then $\mathcal{A}(r)$ is a $C^{1+}$ bounded atlas with bounded geometry.

Proof. Suppose that $I$ and $J$ are either both $\iota$-leaf $n$-cylinders or else that one of them is and the other is an $\iota$-leaf $n$-gap. In addition, suppose that they have a common endpoint. Consider the set of ratios $r(I: J)$. By compactness and continuity, when we restrict $n$ to be 1 , then the set $S$ of such ratios is bounded away from 0 and $\infty$. However, since $r$ is $f$-invariant, all other such ratios $r(I: J)$ are in this set $S$. This also implies that for all endpoints $x$ and $y$ of an $\iota$-leaf $n$-cylinder or $\iota$-leaf $n$-gap $I$, we have that $|I|_{i} \leq \mathcal{O}\left(\left(d_{\Lambda}(x, y)\right)^{\beta}\right)$ and $d_{\Lambda}(x, y) \leq \mathcal{O}\left(|I|_{i}^{\beta}\right)$, for some $0<\beta<1$ independent of $i, I$ and $R$.

Proposition 3.4. Let $r$ be an ı-ratio function. Then inequality (3.2) implies the following inequality: there is a $0<\alpha \leq 1$ such that for every basic holonomy $\theta: I \rightarrow J$ defined with respect to the rectangle $R$,

$$
\left|\log \frac{r\left(\theta I_{1}: \theta I_{2}\right)}{r\left(I_{1}: I_{2}\right)}\right| \leq \mathcal{O}\left(\left(d_{\Lambda}(I, J)|K|\right)^{\alpha}\right)
$$

for all c-leaf segments $I_{1}, I_{2} \subset K$ in $I$. Here for $|K|$ one takes $r(K: \ell(\xi, R))$ which is its length measured in a chart of the bounded atlas $\mathcal{A}(r)$, where $\xi \in K$. The constant $\alpha$ depends only upon $r$ and the constant of proportionality depends only upon $r$ and $R$.

Proof. Take the largest $n$ such that the $\iota$-leaf segments $I_{1}$ and $I_{2}$ are contained in the union of two $n$-cylinders with a common endpoint. By inequality (3.2) and since the ratio functions are $f$-invariant, we have

$$
\begin{aligned}
\left|\log \frac{r\left(\theta I_{1}: \theta I_{2}\right)}{r\left(I_{1}: I_{2}\right)}\right| & =\left|\log \frac{r\left(f_{l}^{-n} \theta I_{1}: f_{l}^{-n} \theta I_{2}\right)}{r\left(f_{l}^{-n} I_{1}: f_{l}^{-n} I_{2}\right)}\right| \\
& \leq \mathcal{O}\left(\left(d_{\Lambda}\left(f_{l}^{-n} I, f_{l}^{-n} J\right)\right)^{\alpha}\right) .
\end{aligned}
$$

By bounded geometry, there are $0<v<1$ and $0<\beta \leq 1$ such that

$$
d_{\Lambda}\left(f_{\iota}^{-n}(I), f_{\iota}^{-n}(J)\right) \leq \mathcal{O}\left(d_{\Lambda}(I, J) v^{n}\right) \leq \mathcal{O}\left(d_{\Lambda}(I, J)|K|^{\beta}\right) .
$$

PROPOSITION 3.5. Suppose that $r$ is an l-ratio function. Then for some $0<\alpha<1, \mathcal{A}(r)$ is a $C^{1+\alpha}$ bounded atlas with bounded geometry and is $C^{1+\alpha}$ foliated. Moreover, there is $0<\beta<1$ such that if $\theta: I \rightarrow J$ is an $\iota$-basic holonomy defined with respect to the rectangle $R$ then for all segments $I_{1}, I_{2} \subset K$ in $I$,

$$
\left|\log \frac{r_{j}\left(\theta I_{1}: \theta I_{2}\right)}{r_{i}\left(I_{1}: I_{2}\right)}\right| \leq \mathcal{O}\left(\left(d_{\Lambda}(I, J)\right)^{\beta}|K|_{i}^{\beta}\right),
$$


where $i: I \rightarrow \mathbb{R}$ and $j: J \rightarrow \mathbb{R}$ are in $\mathcal{A}(r)$ and the constant of proportionality in the $\mathcal{O}$ term only depends upon the choice of $\mathcal{A}(r)$ and upon the rectangle $R$.

Proof. By Proposition 3.3, $\mathcal{A}(r)$ is a $C^{1+\alpha}$ bounded atlas. Inequality (3.5) follows from Proposition 3.4 and so by Proposition 2.5 the holonomies are $C^{1+\alpha}$ smooth for some $0<\alpha<1$. Therefore, $\mathcal{L}(r)$ is a $C^{1+\alpha}$ foliated lamination structure.

3.2. The HR structure associated with a $C^{r}$ hyperbolic realization. Let $\phi$ be a $C^{r}$ hyperbolic realization of $(f, \Lambda, \mathcal{W})$. This determines a unique HR structure as follows.

Let $\mathcal{A}_{\phi}^{\iota}$ and $\mathcal{A}(\rho)^{\iota}$ be the $C^{1+\alpha}$-foliated lamination atlases associated with $\phi$ and with a $C^{1+\gamma}$ Riemannian metric $\rho$ on $M$ (see $\S 2.5$ ). If $I$ is an $\iota$-leaf segment then by $|I|_{\rho}$ we mean the length in the Riemannian metric $\rho$ of the minimal full $\iota$-leaf segment containing $I$.

Before proceeding to the construction of the HR structure associated to $\phi$, we consider the following lemma.

LEMMA 3.6. Let $\rho$ be a $C^{1+\gamma}$ Riemannian metric as previously described. Then for all ı-leaf segments $I$ and $J$ with a common endpoint and for all $n \geq 0$ the following limit exists and is achieved exponentially fast,

$$
r_{\rho}^{\iota}(I: J)=\lim _{n \rightarrow \infty} \frac{\left|f_{l}^{-n} I\right|_{\rho}}{\left|f_{l}^{-n} J\right|_{\rho}} \in \frac{|I|_{\rho}}{|J|_{\rho}}\left(1 \pm \mathcal{O}\left(|I \cup J|_{\rho}^{\gamma}\right)\right),
$$

where the constant of proportionality in the $\mathcal{O}$ term only depends upon the choice of the Riemannian metric $\rho$.

Proof. Let $\hat{I}$ and $\hat{J}$ be the minimal full $\iota$-leaf segments such that $I=\hat{I} \cap \Lambda$ and $J=\hat{J} \cap \Lambda$. Also let $k_{n}: f_{l}^{-n}(\hat{I} \cup \hat{J}) \rightarrow \mathbb{R}$ be an isometry between the Riemannian metric on the full $\iota$-leaf segments and the Euclidean metric on the reals.

The maps $\hat{f}_{n}: k_{n} \circ f_{\imath}^{-n}(\hat{I} \cup \hat{J}) \rightarrow k_{n+1} \circ f_{\iota}^{-(n+1)}(\hat{I} \cup \hat{J})$ defined by $\hat{f}_{n}=k_{n+1} \circ f_{\iota}^{-1} \circ k_{n}$ are $C^{1+\gamma}$ and have $C^{1+\gamma}$ norm uniformly bounded for all $n \geq 0$. Hence, by the Mean Value Theorem and by the hyperbolicity of $\Lambda$ for $f$, we get

$$
\begin{aligned}
\left|\frac{\left|f_{l}^{-n} I\right|_{\rho}}{\left|f_{l}^{-n} J\right|_{\rho}} \frac{|J|_{\rho}}{|I|_{\rho}}\right| & \leq \sum_{i=0}^{n-1}\left|\log \hat{f}_{i}^{\prime}\left(x_{i}\right)-\log \hat{f}_{i}^{\prime}\left(y_{i}\right)\right| \\
& \leq \mathcal{O}\left(|I \cup J|_{\rho}^{\gamma}\right),
\end{aligned}
$$

where $x_{i} \in k_{i} \circ f_{l}^{-i} \hat{I}$ and $y_{i} \in k_{i} \circ f_{l}^{-i} \hat{J}$, so (3.6) follows.

PROPOSITION 3.7. Let $\phi$ be a $C^{1+}$ hyperbolic realization of $(f, \Lambda, \mathcal{W})$ in $M$. There is a unique $H R$ structure $H R_{\phi}=\left(r^{s}, r^{u}\right)$ on $\Lambda$ such that the $C^{1+}$ stable and unstable foliated lamination atlases $\mathcal{A}_{\phi}^{s}$ and $\mathcal{A}_{\phi}^{u}$ induced by the realization $\phi$ have the following property:

(*) for $\iota=s$ and $u$, a map $i: I \rightarrow \mathbb{R}$ defined on an $\iota$-leaf segment I is $C^{1+\alpha}$-compatible with all $j \in \mathcal{A}\left(r^{\iota}\right)$ if and only if it is $C^{1+\alpha}$-compatible with all $j \in \mathcal{A}_{\phi}^{\iota}$.

Furthermore, $\left(r^{s}, r^{u}\right)=\left(r_{\rho}^{s}, r_{\rho}^{u}\right)$, for any $C^{1+\gamma}$ Riemannian metric $\rho$. 
Proof. Let us start proving that $r_{\rho}^{\iota}$ is an $\iota$-ratio function. By construction (see (3.6)), we obtain that $r_{\rho}^{l}$ is continuous, satisfies (3.1) and is invariant under $f$. So, it is enough to prove that $r_{\rho}^{\iota}$ satisfies (3.2).

Let $\theta: I \rightarrow J$ be a basic $\iota$-holonomy. Let $n$ be the integer part of $\left(\log d_{\Lambda}(I, J)\right) /$ $(2 \log 2)$. Let $\hat{\theta}: f_{l}^{-n} I \rightarrow f_{\iota}^{-n} J$ be the basic $\iota$-holonomy given by $\hat{\theta}(x)=f_{l}^{-n} \circ \theta \circ f_{l}^{n}(x)$. By the $f$ invariance of $r_{\rho}^{\iota}$, for all $\iota$ leaf segments $I_{1}, I_{2} \subset K$ in $I$, we have that

$$
\left|\log \frac{r\left(\theta I_{1}: \theta I_{2}\right)}{r\left(I_{1}: I_{2}\right)}\right|=\left|\log \frac{r\left(\hat{\theta} f_{l}^{-n} I_{1}: \hat{\theta} f_{l}^{-n} I_{2}\right)}{r\left(f_{l}^{-n} I_{1}: f_{l}^{-n} I_{2}\right)}\right| .
$$

By (3.6) and bounded geometry, there is $0<\beta_{1} \leq 1$, such that

$$
\begin{aligned}
\left|\log r\left(\hat{\theta} f_{l}^{-n} I_{1}: \hat{\theta} f_{\iota}^{-n} I_{2}\right) \frac{\left|f_{l}^{-n} \hat{\theta} I_{2}\right|_{\rho}}{\left|f_{l}^{-n} \hat{\theta} I_{1}\right|_{\rho}}\right| & \leq \mathcal{O}\left(\left|f_{\iota}^{-n} \hat{\theta} I\right|_{\rho}^{\gamma}\right) \leq \mathcal{O}\left(2^{-n \gamma \beta_{1}}\right) \\
& \leq \mathcal{O}\left(d_{\Lambda}(I: J)^{\gamma \beta_{1} / 2}\right) .
\end{aligned}
$$

Similarly, we have

$$
\left|\log r\left(\hat{\theta} f_{\iota}^{-n} I_{2}: \hat{\theta} f_{\iota}^{-n} I_{1}\right) \frac{\left|f_{l}^{-n} I_{1}\right|_{\rho}}{\left|f_{\iota}^{-n} I_{2}\right|_{\rho}}\right| \leq \mathcal{O}\left(d_{\Lambda}(I: J)^{\gamma \beta_{1} / 2}\right) .
$$

By Theorem 2.2 in [14], the basic $\iota$-holonomies satisfy (2.2) and so (2.3), with respect to the charts in the lamination atlas $\mathcal{A}^{\iota}(\rho)$. Hence, for some $0<\beta_{2} \leq 1$, we have

$$
\begin{aligned}
\left|\log \frac{\left|f_{l}^{-n} \hat{\theta} I_{1}\right|_{\rho}}{\left|f_{l}^{-n} \hat{\theta} I_{2}\right|_{\rho}} \frac{\left|f_{l}^{-n} I_{2}\right|_{\rho}}{\left|f_{l}^{-n} I_{1}\right|_{\rho}}\right| & \leq \mathcal{O}_{R}\left(d_{\Lambda}\left(f_{l}^{-n} I, f_{l}^{-n} J\right)^{\beta_{2}}\right) \\
& \leq \mathcal{O}_{R}\left(d_{\Lambda}(I, J)^{\beta_{2} / 2}\right) .
\end{aligned}
$$

Applying (3.8), (3.9) and (3.10) to (3.7), we obtain

$$
\left|\log \frac{r\left(\theta I_{1}: \theta I_{2}\right)}{r\left(I_{1}: I_{2}\right)}\right| \leq \mathcal{O}\left(d_{\Lambda}(I: J)^{\beta_{3}}\right),
$$

where $\beta_{3}=\min \left\{\gamma \beta_{1} / 2, \beta_{2} / 2\right\}$. Thus, $r_{\rho}^{\iota}$ satisfies (3.2), and so is an $\iota$-ratio function.

Let us prove that $r_{\rho}^{\iota}$ satisfies property $(*)$. As observed in $\$ 2.5$, the overlap maps between charts in $\mathcal{A}_{\phi}^{l}$ and $\mathcal{A}^{l}(\rho)$ are $C^{1+}$ compatible. Hence, it is enough to prove that the overlap map between the charts $i: I \rightarrow \mathbb{R}$ in $\mathcal{A}^{l}\left(r_{\rho}\right)$ and the charts $j: I \rightarrow \mathbb{R}$ in $\mathcal{A}^{\iota}(\rho)$ are $C^{1+}$ compatible. By (3.6), for all $\iota$ leaf segments $I_{1}, I_{2} \subset K$ in $I$, we have

$$
\left|\log \frac{\left|I_{1}\right|_{i}}{\left|I_{2}\right|_{i}} \frac{\left|I_{2}\right|_{j}}{\left|I_{1}\right|_{j}}\right|=\left|\log r\left(I_{1}: I_{2}\right) \frac{\left|I_{2}\right|_{\rho}}{\left|I_{1}\right|_{\rho}}\right| \leq \mathcal{O}\left(|K|_{i}^{\gamma}\right) .
$$

Hence, the overlap map (or identity map) between the charts $i$ and $j$ satisfies (2.1), taking in (2.1) the holonomy map $\theta$ equal to the identity map, and so the overlap map has a $C^{1+}$ extension to $\mathbb{R}$ proving property (*).

The uniqueness of the HR structure follows from the $f$-invariance of $r_{\rho}^{s}$ and $r_{\rho}^{u}$ because two HR structures that are compatible with the lamination structures have arbitrarily close ratios on sufficiently small segments and therefore, since the ratios are $f$-invariant, they must be the same. 


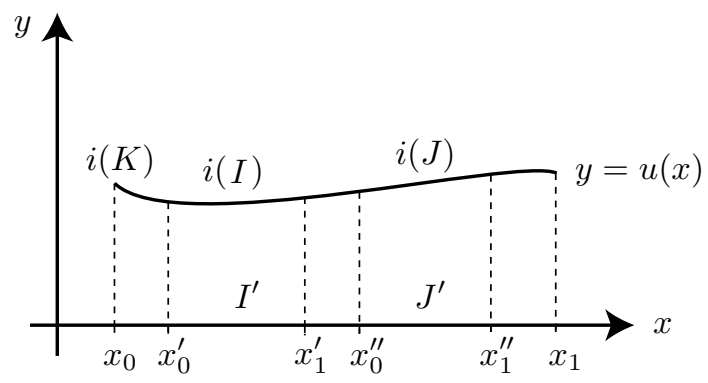

FIGURE 2. The images of $I$ an $J$ by $i$ and their projections in the horizontal axis.

We now consider some simple results that show that the ratio functions can be defined in other natural ways and enable us to prove that the HR structure is an invariant of $C^{1+}$ conjugacy.

LEMMA 3.8. Fix a bounded atlas for the $C^{1+\gamma}$ structure on $M$. Suppose that $I, J$ and $K$ are full -leaf segments with $I, J \subset K$ and that in some chart $i$ of the atlas, $K$ has the form $y=u(x)$ with $x \in\left(x_{0}, x_{1}\right)$ and $u^{\prime}(x)=0$ for some $x \in\left(x_{0}, x_{1}\right)$. Let $I^{\prime}=\left\{(x, 0): x_{0}^{\prime}<x<x_{1}^{\prime}\right\}$ and $\left\{(x, 0): x_{0}^{\prime \prime}<x<x_{1}^{\prime \prime}\right\}$ be, respectively, the projection of $i(I)$ and $i(J)$ onto the $x$-axis and let $I^{\prime \prime}=i^{-1}\left(I^{\prime}\right)$ (see Figure 2). Then there is $0<\alpha<1$ such that

$$
\begin{aligned}
& \frac{|I|_{\rho}}{\left|I^{\prime \prime}\right|_{\rho}} \in 1 \pm O\left(|K|_{\rho}^{\alpha}\right) \\
& \frac{|I|_{\rho}}{|J|_{\rho}} \in\left(1 \pm O\left(|K|_{\rho}^{\alpha}\right)\right) \frac{\left|x_{1}^{\prime}-x_{0}^{\prime}\right|}{\left|x_{1}^{\prime \prime}-x_{0}^{\prime \prime}\right|} \\
& \frac{|I|_{\rho}}{|J|_{\rho}} \in\left(1 \pm O\left(|K|_{\rho}^{\alpha}\right)\right) \frac{\|i(I)\|}{\|i(J)\|}
\end{aligned}
$$

where $\|i(I)\|$ and $\|i(J)\|$ are, respectively, the Euclidean distances between the endpoints of $i(I)$ and $i(J)$. In each case the constants of proportionality only depend upon the atlas, $\rho$ and the $C^{1+\gamma}$ norm of $u$ and $\alpha$ only depends upon the atlas.

Proof. Since $\rho$ is $C^{1+\gamma}$ we can assume that in each chart of the atlas it can be written in the form $g_{11} d x^{2}+g_{12} d x d y+g_{22} d y^{2}$ where the $g_{i j}$ are $C^{\gamma}$ with uniformly bounded $C^{\gamma}$ norm. Then integrating $\rho$ along $y=u(x)$ and $y=0$, and using that $\left|u^{\prime}\right|$ is uniformly bounded, we get

$$
|I|_{\rho},\left|I^{\prime \prime}\right|_{\rho} \in\left(1 \pm \mathcal{O}\left(|K|_{\rho}^{\alpha}\right)\right) \sqrt{g_{11}\left(x_{0}\right)}\left|x_{1}^{\prime}-x_{0}^{\prime}\right| .
$$

Using the Euclidean metric in $\mathbb{R}^{2}$, a similar argument applies to the line segment with endpoints $\left(x_{0}^{\prime}, u\left(x_{0}^{\prime}\right)\right)$ and $\left(x_{1}^{\prime}, u\left(x_{1}^{\prime}\right)\right)$ showing that

$$
\|i(I)\| \in\left(1 \pm \mathcal{O}\left(|K|_{\rho}^{\alpha}\right)\right)\left|x_{1}^{\prime}-x_{0}^{\prime}\right| .
$$

Similarly for $J$. Hence, (3.11) follows from combining these results. 
Proposition 3.9. If $\psi$ is a $C^{1+}$ hyperbolic realization of $(f, \Lambda, \mathcal{W})$ in $M$ which is $C^{1+}$ conjugated to $\phi$ then $H R_{\psi}=H R_{\phi}$.

Proof. Suppose that $\varphi$ and $\psi$ are $C^{1+\beta}$ conjugated. Then by conjugating $\psi$ with the conjugacy we obtain a new diffeomorphism $\psi^{\prime}$ that has the same invariant set $\Lambda$ as $\varphi$ and for which $\varphi\left|\Lambda=\psi^{\prime}\right| \Lambda$. Moreover, it is clear that the HR structures of $\psi$ and $\psi^{\prime}$ are the same since the conjugacy maps the full $\iota$-leaf segments of $\psi$ to the full $\iota$-leaf segments of $\psi^{\prime}$. Thus we replace $\psi$ by $\psi^{\prime}$ and assume that $\psi$ has the properties of $\psi^{\prime}$. In particular, this means that $K$ is a $\iota$-leaf segment for $\varphi$ if and only if it is one for $\psi$. In this case we denote by $K_{\varphi}$ and $K_{\psi}$ the minimal full $\iota$-leaf segments containing $K$ for $\varphi$ and $\psi$ respectively. Moreover, without loss of generality we may assume that $\alpha=\beta=\gamma$, where $\alpha$ and $\gamma$ are as in Lemma 3.8.

Since $\Lambda$ is hyperbolic and compact it can be covered by a finite set of charts of $M$ such that in each chart both the full $u$-leaf segments $K_{\varphi}$ and $K_{\psi}$ are plots of the form $y=u(x)$ where $u$ is $C^{1+\gamma}$ and has a uniformly bounded $C^{1+\gamma}$ norm.

Thus for a chart containing $K_{\varphi}$ and $K_{\psi}, K_{\varphi}$ is of the form $y=u_{\varphi}(x)$ with $x \in\left(x_{0}, x_{1}\right)$. Then by a composition of this chart with a transformation of the form $(x, y) \rightarrow(x, y-$ $\left.u_{\varphi}(x)\right)$ we obtain a chart $i=i_{K}$ in which $i\left(K_{\varphi}\right)$ is of the form $\left\{(x, 0): x_{0}<x<x_{1}\right\}$. The set of such charts $\left\{i_{K}\right\}$ is a bounded $C^{1+\gamma}$ atlas, since the maps $u$ have a uniformly bounded $C^{1+\gamma}$ norm.

Now consider the image of $K_{\psi}$ under $i=i_{K}$. Recall that $K_{\varphi} \cap K_{\psi}=K \subset \Lambda$ and that each point of $K$ is an accumulation point. Therefore $K_{\varphi}$ and $K_{\psi}$ are tangential at all points of $K$. Thus in a neighbourhood $U$ of $i(K), K_{\psi}$ is of the form $y=u_{\psi}(x)$ where $u_{\psi}(x)=0$ and $u_{\psi}^{\prime}(x)=0$ if $x \in K$. We can now apply Lemma 3.8 to deduce that

$$
\frac{\left|I_{\varphi}\right|_{\rho}}{\left|J_{\varphi}\right|_{\rho}} \in\left(1 \pm O\left(|K|_{\rho}^{\gamma}\right)\right) \frac{\left|I_{\psi}\right|_{\rho}}{\left|J_{\psi}\right|_{\rho}} .
$$

From this it follows immediately that the $\iota$-ratio functions are the same for $\varphi$ and $\psi$, and hence that they induce the same HR structures.

Lemma 3.10. Fix a bounded atlas for the $C^{1+\gamma}$ structure on $M$. Suppose that $I, J$ and $K$ are full $\mathrm{l}$-leaf segments with $I, J \subset K$ then

$$
\frac{|I|_{\rho}}{|J|_{\rho}} \in\left(1 \pm O\left(|K|_{\rho}^{\gamma}\right)\right) \frac{\|i(I)\|}{\|i(J)\|}
$$

where $i$ is any chart in the atlas which contains $K$ in its domain and $\|i(I)\|$ and $\|i(J)\|$ are, respectively, the Euclidean distances between the endpoints of $i(I)$ and $i(J)$. The constants of proportionality depend only upon the atlas $\rho$ and the bounded atlas considered.

Proof. Consider a chart $i$ whose domain contains $K$. After composing $i$ with a rotation and a translation if necessary we obtain that if $K$ is sufficiently small then $i(K)$ is of the form $y=u(x)$ with $x \in\left(x_{0}, x_{1}\right)$ and $u\left(x_{0}\right)=0=u\left(x_{1}\right)$ where the $C^{1+\gamma}$ norm of $u$ is uniformly bounded. The result then follows directly from Lemma 3.8. 
COROllary 3.11. Suppose that instead of using equation (3.6) to define the ratios $r(I: J)$ we use the Euclidean distances so that

$$
r_{e}^{\iota}(I: J)=\lim _{n \rightarrow \infty} \frac{\left\|i f_{l}^{n} I\right\|}{\left\|i f_{l}^{n} J\right\|}
$$

where $\left\|i f_{\iota}^{n} I\right\|$ and $\left\|i f_{\imath}^{n} J\right\|$ are as in Lemma 3.8. Then $\left(r_{e}^{s}, r_{e}^{u}\right)=\left(r_{\rho}^{s}, r_{\rho}^{u}\right)$.

\section{Orthogonal structures}

In this section $r=k+\alpha$ where $k \geq 1$ is an integer and $0<\alpha \leq 1$. An orthogonal chart $(i, U)$ on $\Lambda$ is an embedding $i: U \rightarrow \mathbb{R}^{2}$ of an open subset $U$ of $\Lambda$ which embeds every leaf segment in $U$ into a horizontal or vertical arc of $\mathbb{R}$ (say stables into horizontals and unstables into verticals). Two such charts $\left(i_{1}, U_{1}\right)$ and $\left(i_{2}, U_{2}\right)$ on $\Lambda$ are $C^{r}$ compatible if the chart overlap map $i_{2} \circ i_{1}^{-1}: i_{1}\left(U_{1} \cap U_{2}\right) \rightarrow i_{2}\left(U_{1} \cap U_{2}\right)$ is $C^{r}$ in the sense that it extends to a $C^{r}$ diffeomorphism of a neighbourhood of $i_{1}\left(U_{1} \cap U_{2}\right)$ in $\mathbb{R}^{2}$ onto a neighbourhood of $i_{2}\left(U_{1} \cap U_{2}\right)$ in $\mathbb{R}^{2}$.

Definition 4.1. A $C^{r}$ orthogonal atlas $\mathcal{O}$ on $\Lambda$ is a set of orthogonal charts which cover $\Lambda$ and are $C^{r}$ compatible with each other. Such an atlas is said to be bounded if its overlap maps have a uniformly bounded $C^{r}$ norm, with the bound depending only upon the atlas $\mathcal{O}$.

Since $\Lambda$ is compact any atlas contains a bounded atlas.

Proposition 4.2. An HR structure on $\Lambda$ canonically determines a $C^{1+}$ bounded orthogonal atlas such that for every $x \in \Lambda$ there is a chart $\left(i_{x}, U_{x}\right)$ with $x \in U_{x}$ with the following properties: ( $i$ ) the image by $i_{x}$ of the l-leaf segments passing through $x$ determines the same affine structure on these leaf segments as the one given by the HR structure; and (ii) the map $i_{f(x)} \circ f \circ i_{x}^{-1}$ has an affine extension to $\mathbb{R}^{2}$ (in particular is $C^{1+}$ ).

Proof. Given an HR structure on $\Lambda$ we construct orthogonal charts using small rectangles $R$ as follows. Suppose that $x \in \Lambda$ and $R$ is a small rectangle containing $x$. Let $\ell^{s}(x, R)$ and $\ell^{u}(x, R)$ denote the stable and unstable leaf segments of $x$ in $R$ with an orientation chosen on them. For $y \in R$, let $i_{s}(y)= \pm r^{s}\left(\ell^{s}(x, y): \ell^{s}(x, R)\right)$ where the plus sign is chosen if $y$ is positively oriented with respect to $x$ and the minus sign otherwise. Define similarly $i_{u}$. The chart $i$ on $R$ is now given by $i_{x}(z)=\left(i_{s}([z, x]), i_{u}([x, z])\right) \in \mathbb{R}^{2}$. Hence, this chart satisfies property (i) of this proposition.

Since the HR structure determines an affine structure along leaf segments which is kept invariant by $f$, for every $x \in \Lambda$ the map $i_{f(x)} \circ f \circ i_{x}^{-1}$ has a smooth (affine) extension to $\mathbb{R}^{2}$.

Since an HR structure determines a unique affine structure on all leaf segments and since the basic holonomies for this are $C^{1+\alpha}$ by Proposition 3.5 for some $\alpha>0$, the overlap map between any two canonical charts $i_{x}$ and $i_{y}$ has a $C^{1+}$ extension (not necessarily unique). Therefore, the orthogonal charts form a $C^{1+}$ orthogonal atlas.

The converse statement follows in a similar way to the proof of Proposition 3.7. 
5. HR structures determine smooth conjugacy classes

As we have seen, a $C^{1+}$ hyperbolic realization $\phi$ of $(f, \Lambda, \mathcal{W})$ determines an HR structure which is an invariant of the $C^{1+}$ conjugacy class of $\phi$. In Proposition 4.2, for any given HR structure we have constructed a corresponding $C^{1+}$ orthogonal atlas $\mathcal{O}$. Using $\mathcal{O}$, we will prove in Proposition 5.5 that an HR structure is a complete invariant of the $C^{1+}$ conjugacy class of $\phi$. In Proposition 5.7, again using the $C^{1+}$ orthogonal atlas, we will construct for any given HR structure $\left(r^{s}, r^{u}\right)$ a $C^{1+}$ hyperbolic realization $\phi$ of $(f, \Lambda, \mathcal{W})$ with respect to a $C^{1+}$ structure $\mathcal{S}_{\mathrm{HR}}$ such that $r_{\phi}^{s}=r^{s}$ and $r_{\phi}^{u}=r^{u}$. Putting together Propositions 3.7, 5.5 and 5.7 we obtain the following theorem.

THEOREM 5.1. Given $(f, \Lambda, \mathcal{W})$, there is a natural one-to-one correspondence

$$
(\text { HR structures }) \longleftrightarrow\left(\begin{array}{c}
C^{1+} \text { conjugacy } \\
\text { classes }
\end{array}\right)
$$

Definition 5.2. A structure $\mathcal{S}_{\phi}$ of a $C^{r}$ hyperbolic realization $\phi$ is holonomically optimal if it maximizes the smoothness of the holonomy maps amongst the systems in the $C^{1+}$ conjugacy class of $\phi$.

At the end of this section we will prove the following optimality result.

THEOREM 5.3. (i) The structure $\mathcal{S}_{\mathrm{HR}}$ determined by an orthogonal atlas $\mathcal{O}$ is the holonomically optimal representative of the $C^{1+}$ conjugacy class.

(ii) If $\phi$ and $\psi$ are $C^{r}$ Anosov diffeomorphisms determining the same HR structure then they are $C^{r-\varepsilon}$ conjugated for all small $\varepsilon>0$ where $r>1$.

Proposition 5.4. Let $\phi$ be a $C^{1+}$ hyperbolic realization of $(f, \Lambda, \mathcal{W})$ with an associated structure $\mathcal{S}_{\phi}$ and let $\mathcal{O}$ denote the orthogonal atlas determined by $H R_{\phi}$. For every orthogonal chart $i: R \rightarrow \mathbb{R}^{2}$ in $\mathcal{O}$ and for every $z \in R$ there is a chart $(v, V) \in \mathcal{S}_{\phi}$ with $z \in V$ such that $V \cap \Lambda=V \cap R$ and such that $v|(V \cap \Lambda)=i|(V \cap R)$.

Proof. Let $i: R \rightarrow \mathbb{R}^{2}$ be an orthogonal chart and $\left(j, V^{\prime}\right)$ be a chart of $\mathcal{S}_{\phi}$ such that (i) each full $s$-leaf segment and each full $u$-leaf segment in $U$ are the graph of a $C^{1+}$ function respectively over the $x$ - and $y$-axes; (ii) $j(z)=0$; and (iii) $j \circ i^{-1}$ is the identity along the leaf segments $\ell^{s}(z, R)$ and $\ell^{u}(z, R)$. Thus $j \circ i^{-1}(0)=0$. Let $K=i(R)$, and the map $u: K \rightarrow \mathbb{R}^{2}$ be defined by $u=j \circ i^{-1}$. We are going to prove that $u$ has a $C^{1+}$ extension $\tilde{u}: \mathbb{R}^{2} \rightarrow \mathbb{R}^{2}$ and that the derivative $d \tilde{u}(0)$ of $\tilde{u}$ at 0 is an isomorphism. Thus, there is a small open set $V \subset V^{\prime}$ containing $z$ such that $V \cap \Lambda=V \cap R$ and such that $\tilde{u} \mid j(V)$ is a $C^{1+}$ diffeomorphism onto its image. Hence, $\left(v=\tilde{u}^{-1} \circ j, V\right)$ is a chart $C^{1+}$ compatible with the structure $\mathcal{S}_{\phi}$ and $v|(V \cap \Lambda)=i|(V \cap R)$. To prove that $u$ has a $C^{1+}$ extension $\tilde{u}: \mathbb{R}^{2} \rightarrow \mathbb{R}^{2}$ we start by finding the natural candidates $\partial_{x} u(x, y)$ and $\partial_{y} u(x, y)$ to be the derivatives $\partial_{x} \tilde{u}(x, y)$ and $\partial_{y} \tilde{u}(x, y)$ of the extension $\tilde{u}$ at the points $(x, y) \in K$.

Let $\pi_{s}: \mathbb{R}^{2} \rightarrow \mathbb{R}$ and $\pi_{u}: \mathbb{R}^{2} \rightarrow \mathbb{R}$ be the projections onto the $x$ - and $y$-axes, respectively. For every $(0, y) \in K$, consider the $s$-spanning leaf segments $I_{y}^{s}$ in $R$ of the form $\left(x, v_{y, s}(x)\right)$ for $x \in \pi_{s} \circ i\left(I_{y}^{s}\right)$ in this chart, and for every $(x, 0) \in K$, consider the $u$-spanning leaf segments $I_{y}^{u}$ in $R$ of the form $\left(v_{x, u}(y), y\right)$ for $y \in \pi_{u} \circ i\left(I_{s}^{u}\right)$ in this chart, where $v_{y, s}$ and $v_{x, u}$ are $C^{1+}$ functions. Consider the basic holonomies $\theta_{z, \iota}: I_{0}^{\iota} \rightarrow I_{z}^{\iota}$ in 


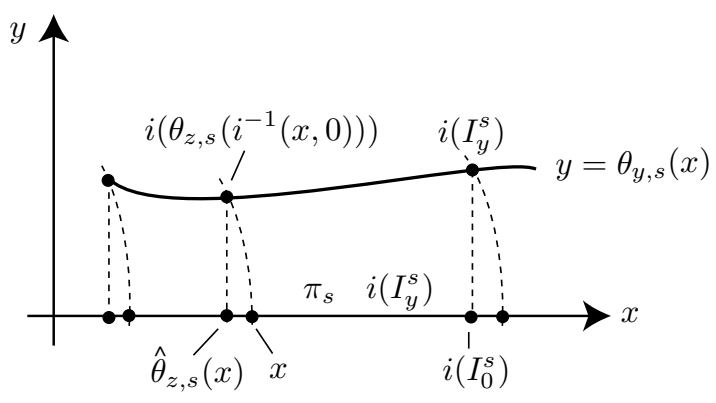

FIGURE 3. The map $\hat{\theta}_{z, l}$.

$R$ and let $\hat{\theta}_{z, \iota}: \pi_{\iota} \circ i\left(I_{0}^{\iota}\right) \subset \mathbb{R} \rightarrow \mathbb{R}$ be defined by $\hat{\theta}_{z, \iota}(x)=\pi_{\iota} \circ i \circ \theta_{z, \iota} \circ i^{-1}(x, 0)$ (see Figure 3). Hence,

$$
\begin{aligned}
u(x, y) & =\left(\hat{\theta}_{y, s}(x), v_{y, s}\left(\hat{\theta}_{y, s}(x)\right)\right) \\
& =\left(v_{x, u}\left(\hat{\theta}_{x, u}(y)\right), \hat{\theta}_{x, u}(y)\right) .
\end{aligned}
$$

By Corollary 2.4 in [14], the maps $\hat{\theta}_{y, s}$ and $\hat{\theta}_{x, u}$ have $C^{1+\alpha_{1}}$ extensions $\tilde{\theta}_{y, s}$ and $\tilde{\theta}_{x, u}$ which vary Hölder continuously with $y$ and $x$ respectively, for some $0<\alpha_{1}<1$. Thus, we define

$$
\begin{aligned}
& \partial_{x} u(x, y)=\left(\tilde{\theta}_{y, s}^{\prime}(x), v_{y, s}^{\prime}\left(\tilde{\theta}_{y, s}(x)\right) \tilde{\theta}_{y, s}^{\prime}(x)\right), \\
& \partial_{y} u(x, y)=\left(v_{x, u}^{\prime}\left(\tilde{\theta}_{x, u}(y)\right) \tilde{\theta}_{x, u}^{\prime}(y), \tilde{\theta}_{x, u}^{\prime}(y)\right) .
\end{aligned}
$$

Since $\tilde{\theta}_{y, s}$ and $v_{y, s}$ are $C^{1+}$, for every $y \in \pi_{u} \circ i\left(\ell^{u}(z, R)\right), \partial_{x} u(x, y)$ varies Hölder continuously with $x \in \pi_{s} \circ i\left(\ell^{s}(z, R)\right)$. Since the $C^{1+\alpha_{1}}$ extensions $\tilde{\theta}_{y, s}$ and $\tilde{\theta}_{x, u}$ vary Hölder continuously with $y$ and $x$, and by the Hölder continuity of the stable and unstable bundles (see $\S 6$ in [6]), for every $x \in \pi_{s} \circ i\left(\ell^{s}(z, R)\right), \partial_{x} u(x, y)$ varies Hölder continuously with $y \in \pi_{u} \circ i\left(\ell^{u}(z, R)\right)$. Therefore, $\partial_{x} u(x, y)$ varies Hölder continuously with $(x, y) \in K$. Similarly, we obtain that $\partial_{y} u(x, y)$ varies Hölder continuously with $(x, y) \in K$.

By the Whitney Extension Lemma (see [1]), the map $u$ has a $C^{1+}$ extension $\tilde{u}$ with $\partial_{x} \tilde{u}(x, y)=\partial_{x} u(x, y)$ and $\partial_{y} \tilde{u}(x, y)=\partial_{y} u(x, y)$, if

$$
\left\|U\left((x, y),\left(x+h_{x}, y+h_{y}\right)\right)\right\| \leq \mathcal{O}\left(\left\|\left(h_{x}, h_{y}\right)\right\|^{1+\alpha}\right)
$$

for some $\alpha>0$, where

$$
U\left((x, y),\left(x^{\prime}, y^{\prime}\right)\right)=u\left(x^{\prime}, y^{\prime}\right)-u(x, y)-\partial_{x} u(x, y)\left(x^{\prime}-x\right)-\partial_{y} u(x, y)\left(y^{\prime}-y\right) .
$$

Since $\tilde{\theta}_{y, s}$ and $v_{y, s}$ are $C^{1+}$, for all $y \in \pi_{u} \circ i\left(\ell^{u}(z, R)\right)$, we have that the maps $u_{y}: \pi_{s} \circ i\left(\ell_{\phi}^{s}(z, R)\right) \rightarrow \mathbb{R}^{2}$ defined by $u_{y}(x)=\left(\tilde{\theta}_{y, s}(x), v_{y, s}\left(\tilde{\theta}_{y, s}(x)\right)\right)$ are $C^{1+\alpha_{1}}$, for some $\alpha_{1}>0$. Since $\tilde{\theta}_{x, u}$ and $v_{x, u}$ are $C^{1+}$, for all $x \in \pi_{s} \circ i\left(\ell^{u}(z, R)\right)$, we have that the maps $u_{x}: \pi_{u} \circ i\left(\ell_{\phi}^{u}(z, R)\right) \rightarrow \mathbb{R}^{2}$ defined by $u_{x}(y)=\left(v_{x, u}\left(\tilde{\theta}_{x, u}(y)\right), \tilde{\theta}_{x, u}(y)\right)$ are $C^{1+\alpha_{1}}$, for some $\alpha_{1}>0$. Therefore,

$$
\begin{aligned}
u\left(x+h_{x}, y+h_{y}\right)-u(x, y) & =u_{y+h_{y}}\left(x+h_{x}\right)-u_{y+h_{y}}(x)+u_{x}\left(y+h_{y}\right)-u_{x}(y) \\
& \in \partial_{x} u\left(x, y+h_{y}\right) h_{x}+\partial_{y} u(x, y) h_{y} \pm \mathcal{O}\left(\left\|\left(h_{x}, h_{y}\right)\right\|^{1+\alpha_{1}}\right) .
\end{aligned}
$$


Since $\partial_{x} u(x, y)$ varies Hölder continuously with $(x, y) \in K$, there is a $0<\alpha \leq \alpha_{1}$ such that

$$
\begin{aligned}
U\left((x, y),\left(x+h_{x}, y+h_{y}\right)\right) & \in \partial_{x} u\left(x, y+h_{y}\right) h_{x}-\partial_{x} u(x, y) h_{x} \pm \mathcal{O}\left(\left\|\left(h_{x}, h_{y}\right)\right\|^{1+\alpha_{1}}\right) \\
& \subset \pm \mathcal{O}\left(\left\|\left(h_{x}, h_{y}\right)\right\|^{1+\alpha}\right) .
\end{aligned}
$$

Proposition 5.5. Let $\phi$ and $\psi$ be $C^{1+}$ hyperbolic realizations of $(f, \Lambda, \mathcal{W})$. The maps $\phi$ and $\psi$ are $C^{1+}$ conjugated if and only if they determine the same $H R$ structures on $\Lambda$.

Before proceeding to the proof of this proposition we are going to prove an auxiliary lemma that we will also use later in the proof of Proposition 5.7.

Lemma 5.6. Consider $(f, \Lambda, \mathcal{W})$ and let $h: \Lambda \rightarrow \Lambda$ be a homeomorphism preserving the order along the leaf segments of $\mathcal{W}$. Let $\mathcal{S}$ and $\mathcal{S}^{\prime}$ be $C^{1+}$ structures on $M$ such that there are charts $\left(u_{1}, U_{1}\right), \ldots,\left(u_{p}, U_{p}\right) \in \mathcal{S}$ and $\left(v_{1}, V_{1}\right), \ldots,\left(v_{p}, V_{p}\right) \in \mathcal{S}^{\prime}$ with the following properties:

(i) $\Lambda \subset \bigcup_{q=1}^{p} U_{q}$;

(ii) for every $q=1, \ldots, p, h_{q}: U_{q} \rightarrow V_{q}$ is a $C^{1+}$ diffeomorphism between $\mathcal{S}$ and $\mathcal{S}^{\prime}$ which extends $h \mid\left(\Lambda \cap U_{q}\right)$.

Then $h: \Lambda \rightarrow \Lambda$ extends to a $C^{1+}$ diffeomorphism defined on an open set of $M$.

Let us introduce some useful notions for the proof of this lemma. We say that a rectangle $M_{n}$ is an $\left(N_{s}, N_{u}\right)$-Markov rectangle if, for all $x \in M_{n}$ and for $\iota \in\{s, u\}$, the $\iota$-spanning leaf segments $\ell^{\iota}\left(x, M_{n}\right)$ are $\iota$-leaf $N_{\iota}$-cylinders. Let us consider the set of all $(N, N)$-Markov rectangles $M_{n}$ for some fixed $N>1$. A corner $c$ is an endpoint of a stable spanning segment and of an unstable spanning segment contained in the boundary of an $(N, N)$-Markov rectangle $M_{n}$. A $\iota$-partial side $s$ is a closed $\iota$-leaf segment whose endpoints are corners and such that int $s$ does not contain any corner. Let $\mathcal{C}_{N}$ be the set of all such corners and $\mathcal{S}_{N}$ be the set of all such $s$-partial sides and $u$-partial sides. For all corners $c \in \mathcal{C}_{N}$ and for all partial sides $s \in \mathcal{S}_{N}$, there are corner rectangles $R(c)$ and side rectangles $R(s)$ with the following properties (see Figure 4):

(i) $\quad c \in R(c)$;

(ii) if $c_{1}$ and $c_{2}$ are corners of the $\iota$-partial side $s$ then $s \subset R\left(c_{1}\right) \cup R(s) \cup R\left(c_{2}\right)$ and the $\iota^{\prime}$-boundary of $R(s)$ is contained in $R\left(c_{1}\right) \cup R\left(c_{2}\right)$;

(iii) the rectangles $R(c)$ are pairwise disjoint for all $c \in \mathcal{C}_{N}$;

(iv) the rectangles $R(s)$ are pairwise disjoint for all $s \in \mathcal{S}_{N}$.

Proof. We will consider separately the cases where (i) both the stable and unstable leaf segments are one-dimensional topological manifolds (the Anosov case); (ii) both the stable and unstable leaf segments are Cantor sets (e.g. Smale horseshoes); (iii) the stable leaf segments are Cantor sets and the unstable leaf segments are one-dimensional topological manifolds (attractors); and (iv) the stable leaf segments are one-dimensional topological manifolds and the unstable leaf segments are Cantor sets (repellers).

Case (i). In this case $\Lambda=M$ and so by the hypotheses of this lemma $h: M \rightarrow M$ is a $C^{1+}$ diffeomorphism. 


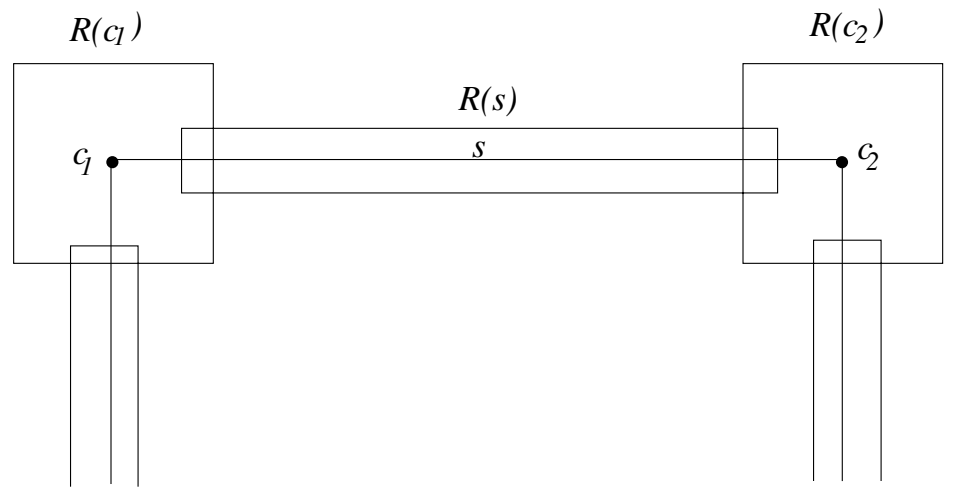

FIGURE 4. The corner and side rectangles.

Case (ii). Since $\Lambda$ is compact and a Cantor set, there is a finite set $\left\{M_{n}: 1 \leq n \leq m\right\}$ of pairwise disjoint rectangles with the following properties: (i) $\bigcup_{n=1}^{m} M_{n} \supset \Lambda$; (ii) for each rectangle $M_{n}$ there are charts $\left(u_{n}, U_{n}\right) \in \mathcal{S}$ and $\left(v_{n}, V_{n}\right) \in \mathcal{S}^{\prime}$ such that $U_{n} \supset M_{n}$ and $h$ has a $C^{1+}$ diffeomorphic extension $h_{n}: U_{n} \rightarrow V_{n}$. Take pairwise disjoint open sets $U_{n}^{\prime} \subset U_{n}$ such that $M_{n} \subset U_{n}^{\prime}$ and the sets $V_{n}^{\prime}=h_{n}\left(U_{n}^{\prime}\right)$ are also pairwise disjoint. The map

$$
\hat{h}: \bigcup_{n=1}^{m} U_{n}^{\prime} \rightarrow \bigcup_{n=1}^{m} V_{n}^{\prime}
$$

defined by $\hat{h} \mid U_{n}^{\prime}=h_{n}$ is a $C^{1+}$ diffeomorphic extension of the conjugacy $h: \Lambda \rightarrow \Lambda$.

Case (iii). Since $\Lambda$ is compact, there exists $N$ large enough such that for every $(N, N)$ Markov rectangle $M_{n}$ there are charts $\left(u_{n}, U_{n}\right) \in \mathcal{S}$ and $\left(v_{n}, V_{n}\right) \in \mathcal{S}^{\prime}$ such that

$$
U_{n} \supset\left(M_{n} \cup\left(\bigcup_{s \in \mathcal{S}_{N} \cap M_{n}} R(s)\right) \cup\left(\bigcup_{c \in \mathcal{C}_{N} \cap M_{n}} R(c)\right)\right)
$$

and $h$ has a $C^{1+}$ diffeomorphic extension $h_{n}: U_{n} \rightarrow V_{n}$. For every corner $c \in \mathcal{C}_{N}$, we choose an $(N, N)$-Markov rectangle $M_{n(c)}$ containing $c$, and an open set $U(c) \supset R(c)$ with the following properties:

(i) for every $(N, N)$-Markov rectangle $M_{m}$ containing $c$,

$$
U(c) \subset U_{m} \quad \text { and } \quad V(c)=h_{n(c)}(U(c)) \subset V_{m} ;
$$

(ii) the sets $U(c)$ are pairwise disjoint for all $c \in \mathcal{C}_{N}$; and

(iii) the sets $V(c)=h_{n(c)}(U(c))$ are also pairwise disjoint for all $c \in \mathcal{C}_{N}$.

We define the $C^{1+}$ diffeomorphic extension

$$
h_{\mathcal{C}}: \bigcup_{c \in \mathcal{C}_{N}} U(c) \rightarrow \bigcup_{c \in \mathcal{C}_{N}} V(c)
$$

of $h \mid\left(\Lambda \cap\left(\bigcup_{c \in \mathcal{C}_{N}} U(c)\right)\right)$ by $h_{\mathcal{C}}\left|U(c)=h_{n(c)}\right| U(c)$. Similarly, for every partial side $s \in \mathcal{S}_{N}$, we choose an $(N, N)$-Markov rectangle $M_{n(s)}$ containing $s$, and an open set $U(s) \supset R(s)$ with the following properties: 


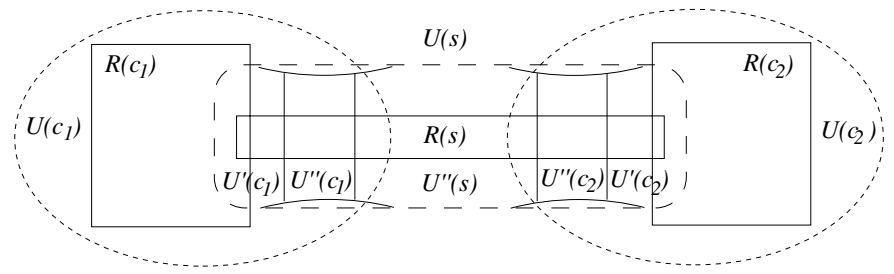

FIGURE 5. The open sets associated with the corner and side rectangles.

(i) for every $(N, N)$-Markov rectangle $M_{m}$ containing $s$,

$$
U(s) \subset U_{m} \quad \text { and } \quad V(s)=h_{n(s)}(U(s)) \subset V_{m} ;
$$

(ii) the sets $U(s)$ are pairwise disjoint for all $s \in \mathcal{S}_{N}$; and

(iii) the sets $V(s)=h_{n(s)}(U(s))$ are also pairwise disjoint for all $s \in \mathcal{S}_{N}$.

We define the $C^{1+}$ diffeomorphic extension

$$
h_{\mathcal{S}}: \bigcup_{s \in \mathcal{S}_{N}} U(s) \rightarrow \bigcup_{s \in \mathcal{S}_{N}} V(s)
$$

of $h \mid\left(\Lambda \cap\left(\bigcup_{s \in \mathcal{S}} U(s)\right)\right)$ by $h_{\mathcal{S}}\left|U(s)=h_{n(s)}\right| U(s)$. Let $s \in \mathcal{S}_{N}$ be a partial side with endpoints $c_{1}$ and $c_{2}$. We define

$$
H_{s}: u_{n(s)}(U(s)) \rightarrow v_{n(s)}(V(s)) \quad \text { and } \quad H_{c_{k}}: u_{n(s)}\left(U\left(c_{k}\right)\right) \rightarrow v_{n(s)}\left(V\left(c_{k}\right)\right)
$$

by

$$
H_{s}=v_{n(s)} \circ h_{\mathcal{S}} \circ u_{n(s)}^{-1} \quad \text { and } \quad H_{c_{k}}=v_{n(s)} \circ h_{\mathcal{C}} \circ u_{n(s)}^{-1}
$$

for $k=1$ and 2. We choose open sets $U^{\prime}(s), U^{\prime}\left(c_{1}\right), U^{\prime}\left(c_{2}\right), U^{\prime \prime}(s)$ and sets $U^{\prime \prime}\left(c_{1}\right)$ and $U^{\prime \prime}\left(c_{2}\right)$ with the following properties (see Figure 5):

(i) $U^{\prime}(s)=U^{\prime}\left(c_{1}\right) \cup U^{\prime \prime}\left(c_{1}\right) \cup U^{\prime \prime}(s) \cup U^{\prime \prime}\left(c_{2}\right) \cup U^{\prime}\left(c_{2}\right)$;

(ii) $s \cap U^{\prime}(s)=s \cap U(s)$;

(iii) $\overline{U^{\prime}\left(c_{1}\right) \cup U^{\prime \prime}\left(c_{1}\right)} \subset U\left(c_{1}\right)$ and $\overline{U^{\prime}\left(c_{2}\right) \cup U^{\prime \prime}\left(c_{2}\right)} \subset U\left(c_{2}\right)$;

(iv) $\overline{U^{\prime \prime}\left(c_{1}\right)} \subset U(s)$ and $\overline{U^{\prime \prime}\left(c_{2}\right)} \subset U(s)$; and

(v) $\overline{U^{\prime}\left(c_{1}\right)} \cap \overline{U^{\prime \prime}(s)}=\emptyset$ and $\overline{U^{\prime}\left(c_{2}\right)} \cap \overline{U^{\prime \prime}(s)}=\emptyset$.


the following properties:

(i) $\tilde{H}_{s} \mid u_{n(s)}\left(U^{\prime \prime}(s)\right)=H_{s}$;

(ii) $\tilde{H}_{s} \mid u_{n(s)}\left(U^{\prime}\left(c_{k}\right)\right)=H_{c_{k}}$ for all $k \in\{1,2\}$; and

(iii) $\tilde{H}_{s}(z)=v_{n(s)} \circ h \circ u_{n(s)}^{-1}(z)$ for all $z \in u_{n(s)}\left(U^{\prime}(s) \cap \Lambda\right)$.

Using the facts that $H_{s}$ and $H_{c_{k}}$ coincide on $u_{n_{s}}\left(U^{\prime}(s) \cap U\left(c_{k}\right) \cap \Lambda\right)$ and that $R(s)$ is compact, there is an open set $\tilde{U}(s) \subset U^{\prime}(s)$ such that $s \cap \tilde{U}(s)=s \cap U^{\prime}(s)$ and such that $\tilde{H}_{s}$ restricted to $u_{n(s)}(\tilde{U}(s))$ is injective. Set $\tilde{V}(s)=v_{n(s)}^{-1} \circ \tilde{H}_{s} \circ u(\tilde{U}(s))$. Letting, for every $c \in \mathcal{C}_{N}, \tilde{U}(c)$ and $\tilde{V}(c)$ be the open sets defined by

$$
\tilde{U}(c)=U(c) \backslash\left(U(c) \cap\left(\bigcup_{s \in \mathcal{S}_{N}} U(s)\right)\right) \quad \text { and } \quad \tilde{V}(c)=h_{\mathcal{C}}(\tilde{U}(c))
$$


we obtain that the map

$$
\tilde{h}:\left(\bigcup_{c \in \mathcal{C}_{N}} \tilde{U}(c)\right) \cup\left(\bigcup_{s \in \mathcal{S}_{N}} \tilde{U}(s)\right) \rightarrow\left(\bigcup_{c \in \mathcal{C}_{N}} \tilde{V}(c)\right) \cup\left(\bigcup_{s \in \mathcal{S}_{N}} \tilde{V}(s)\right)
$$

defined by

$$
\tilde{h}(z)= \begin{cases}v_{n(s)}^{-1} \circ \tilde{H}_{s} \circ u_{n(s)}(z) & \text { for all } z \in \bigcup_{s \in \mathcal{S}_{N}} \tilde{U}(s), \\ h_{\mathcal{C}}(z) & \text { for all } z \in \bigcup_{c \in \mathcal{C}_{N}} \tilde{U}(c),\end{cases}
$$

is a $C^{1+}$ diffeomorphic extension of

$$
h \mid\left(\Lambda \cap\left(\left(\bigcup_{c \in \mathcal{C}_{N}} \tilde{U}(c)\right) \cup\left(\bigcup_{s \in \mathcal{S}_{N}} \tilde{U}(s)\right)\right)\right) .
$$

For any $(N, N)$-Markov rectangle $M_{n}$, letting

$$
\tilde{U}_{n}=\left(\bigcup_{c \in M_{n} \cap \mathcal{C}_{N}} \tilde{U}\left(c_{k}^{n}\right)\right) \cup\left(\bigcup_{s \in M_{n} \cap \mathcal{S}_{N}} \tilde{U}\left(s_{k}^{n}\right)\right)
$$

we have that $\partial M_{n} \subset \tilde{U}_{n}$. We take open sets $\tilde{\tilde{U}}_{n}$ with pairwise disjoint closures and such that $\tilde{\tilde{U}}_{n} \cup \tilde{U}_{n} \supset M_{n}$. Using bump functions, there is a $C^{1+}$ injective map

$$
\hat{H}_{n}: u_{n}\left(\tilde{U}_{n} \cup \tilde{\tilde{U}}_{n}\right) \subset \mathbb{R}^{2} \rightarrow \mathbb{R}^{2}
$$

with the following properties:

(i) $\hat{H}_{n}(z)=v_{n} \circ \tilde{h} \circ u_{n}^{-1}(z)$ for all $z \in u_{n}\left(\tilde{U}_{n} \backslash\left(\tilde{\tilde{U}}_{n} \cap \tilde{U}_{n}\right)\right)$;

(ii) $\hat{H}_{n}(z)=v_{n} \circ h_{n} \circ u_{n}^{-1}(z)$ for all $z \in u_{n}\left(\tilde{\tilde{U}}_{n} \backslash\left(\tilde{\tilde{U}}_{n} \cap \tilde{U}_{n}\right)\right)$; and

(iii) $\hat{H}_{n}(z)=v_{n} \circ h \circ u_{n}^{-1}(z)$ for all $z \in u_{n}\left(\Lambda \cap\left(\tilde{\tilde{U}}_{n} \cup \tilde{U}_{n}\right)\right)$.

Using the fact that $v_{n} \circ \tilde{h} \circ u_{n}^{-1}$ and $v_{n} \circ h_{n} \circ u_{n}^{-1}$ coincide on $u_{n}\left(\Lambda \cap \tilde{U}_{n}\right)$, there is an open set $\hat{U}_{n} \subset \tilde{\tilde{U}}_{n} \cup \tilde{U}_{n}$ containing $M_{n}$ such that $\hat{H}_{n}$ restricted to $u_{n}\left(\hat{U}_{n}\right)$ is injective. Set $\hat{V}_{n}=v_{n}^{-1} \circ \hat{H}_{n} \circ u_{n}\left(\hat{U}_{n}\right)$. Therefore, the map

$$
\hat{h}: \bigcup_{M_{n}} \hat{U}_{n} \rightarrow \bigcup_{M_{n}} \hat{V}_{n}
$$

defined by

$$
\hat{h}(z)=v_{n}^{-1} \circ \hat{H}_{n} \circ u_{n}(z) \text { for all } z \in \hat{U}_{n}
$$

is a $C^{1+}$ diffeomorphism with $\hat{h} \mid \Lambda=h$ which ends the proof of this case.

Case (iv). The proof follows in a similar way to the case (iii).

Proof of Proposition 5.5. By Proposition 3.7, if $\phi$ and $\psi$ are $C^{1+}$ conjugated then they determine the same HR structure on $\Lambda$. Let us prove that if $\phi$ and $\psi$ determine the same HR structure on $\Lambda$ then $\phi$ and $\psi$ are $C^{1+}$ conjugated. Since the HR structures induced by $\phi$ and $\psi$ are the same, for every $z \in \Lambda$ and every rectangle $R$ containing $z$ we have that the orthogonal charts $i: R \rightarrow \mathbb{R}^{2}$ as constructed in Proposition 4.2 are also the same. By Proposition 5.4, for every $z \in \Lambda$ there is an open set $W$ of $M$ and there is an orthogonal chart $i: R \rightarrow \mathbb{R}^{2}$ with the following properties: 
(i) $\quad W \cap R=W \cap \Lambda$;

(ii) $i \mid(W \cap \Lambda)$ extends to a chart $(u, W)$ which is $C^{1+}$ compatible with the structure $\mathcal{S}_{\phi}$; and

(iii) $i \mid(W \cap \Lambda)$ extends to a chart $(v, W)$ which is $C^{1+}$ compatible with the structure $\mathcal{S}_{\psi}$. The identity map id : $\Lambda \rightarrow \Lambda$ is a conjugacy between $\phi$ and $\psi$, and so the map $v \circ$ id $\circ u^{-1} \mid u(W \cap \Lambda)$ is also the identity. Hence, the identity map id $W: u(W) \rightarrow \mathbb{R}^{2}$ is a smooth extension of $v \circ$ id $\circ u^{-1} \mid u(W \cap \Lambda)$. Now, we choose an open set $U \subset W$ of $M$ containing $z$ and such that $u(U) \subset v(W)$. Since $W \cap R=W \cap \Lambda$ we obtain that $U \cap R=U \cap \Lambda$ and by taking $V=v^{-1} \circ \operatorname{id}_{W} \circ u(U)$ we obtain that $V \cap R=V \cap \Lambda$. The map $h_{U}: U \rightarrow V$ is well defined by

$$
h_{U}(z)=v^{-1} \circ \operatorname{id}_{W} \circ u(z)
$$

and is a $C^{1+}$ diffeomorphic extension to $U$ of the conjugacy id|( $\left.\Lambda \cap U\right)$ between $\phi$ and $\psi$. Hence, the hypotheses of Lemma 5.6 are satisfied with $h=$ id $: \Lambda \rightarrow \Lambda$ which implies that the conjugacy id : $\Lambda \rightarrow \Lambda$ between $\phi$ and $\psi$ extends to a $C^{1+}$ diffeomorphism defined on an open set of $M$.

Proposition 5.7. Given an HR structure $\left(r^{s}, r^{u}\right)$ on $\Lambda$, there is a $C^{1+}$ hyperbolic realization $\phi$ of $f$ with the $H R$ structure $H R_{\phi}$ of Proposition 3.7 equal to $\left(r^{s}, r^{u}\right)$.

Proof. Here, we are going to construct a $C^{1+}$ structure $\mathcal{S}_{\mathrm{HR}}$ such that for every $z \in \Lambda$ there is an orthogonal chart $i_{z}: R_{z} \rightarrow \mathbb{R}^{2}$ as constructed in Proposition 4.2 and there is a chart $\left(u_{z}, U_{z}\right) \in \mathcal{S}_{\mathrm{HR}}$ with the following properties:

(i) $\quad z \in R_{z} \cap U_{z}$ and $R_{z} \cap U_{z}=\Lambda \cap U_{z}$; and

(ii) $u_{z}\left|\left(\Lambda \cap U_{z}\right)=i_{z}\right|\left(R_{z} \cap U_{z}\right)$.

Hence, the map $u_{f z} \circ f \circ u_{z}^{-1} \mid\left(R_{z} \cap f^{-1}\left(R_{f z}\right)\right)$ has an affine diffeomorphic extension $F_{z}: \mathbb{R}^{2} \rightarrow \mathbb{R}^{2}$. Taking $U_{z}^{\prime}=u_{z}\left(U_{z}\right) \cap F_{z}^{-1}\left(u_{f z}\left(U_{f z}\right)\right)$ and $V_{z}^{\prime}=F_{z}\left(U_{z}^{\prime}\right)$, we obtain that the map

$$
f_{z}: u_{z}^{-1}\left(U_{z}^{\prime}\right) \rightarrow u_{f z}^{-1}\left(V_{z}^{\prime}\right)
$$

defined by $u_{z}^{-1} \circ F_{z} \circ u_{f z}$ is a $C^{1+}$ diffeomorphic extension of $f \mid\left(\Lambda \cap u_{z}^{-1}\left(U_{z}^{\prime}\right)\right)$ with respect to the $C^{1+}$ structure $\mathcal{S}_{\mathrm{HR}}$. Thus, by compactness of $\Lambda$ and by Lemma 5.6, the map $f: \Lambda \rightarrow \Lambda$ has a $C^{1+}$ diffeomorphic extension $\phi$ to an open set $U_{M}$ of $M$ with respect to the structure $\mathcal{S}_{\mathrm{HR}}$. Let $X^{s}$ be the horizontal axis in $\mathbb{R}^{2}$ and $X^{u}$ be the vertical axis in $\mathbb{R}^{2}$. For every $z \in \Lambda$, we have that $T_{z} M=E_{z}^{s} \oplus E_{z}^{u}$ where $E_{z}^{\iota}=d u_{z}(z)^{-1}\left(X^{\iota}\right)$ for $\iota \in\{s, u\}$. Since $u_{z}\left(\ell^{\iota}\left(z, R_{z}\right) \cap U_{z}\right) \subset X^{\iota}$, we obtain that $d \phi(z)\left(E_{z}^{\iota}\right)=E_{\phi(z)}^{\iota}$. Now, we take a $C^{1+}$ Riemannian metric $\rho$ on $M$ compatible with the $C^{1+}$ structure $\mathcal{S}_{\mathrm{HR}}$. By the bounded geometry of the atlases $\mathcal{A}\left(r^{s}\right)$ and $\mathcal{A}\left(r^{u}\right)$ associated with the HR structure $\left(r^{s}, r^{u}\right)$, there are constants $C>0$ and $\lambda>1$ with the following properties:

(i) $\left|d \phi^{-n}(z) v^{s}\right|_{\rho} \geq C \lambda^{n}\left|v^{s}\right|_{\rho}$ for all $v^{s} \in E_{z}^{s}$; and

(ii) $\left|d \phi^{n}(z) v^{u}\right|_{\rho} \geq C \lambda^{n}\left|v^{u}\right|_{\rho}$ for all $v^{u} \in E_{z}^{u}$.

Therefore, $\phi$ is a hyperbolic realization of $(f, \Lambda, \mathcal{W})$. By the Stable Manifold Theorem, for every $z \in \Lambda$ and for every $\iota \in\{s, u\}$, the leaf $\ell_{\phi}^{\iota}\left(z, R_{z}\right)$ is a $C^{1+}$ manifold. For every triple $(y, z, w)$ of points in $\ell^{\iota}\left(z, R_{z}\right)$, let $\ell^{l}(y, z)$ be the $\iota$-leaf segment with endpoints $y$ and $z$ and $\ell^{\iota}(z, w)$ be the leaf segment with endpoints $z$ and $w$. Applying Lemma 3.8, 
we obtain

$$
\left|\log r^{\iota}(y, z, w) \frac{\left|\ell^{\iota}(z, w)\right|_{\rho}}{\left|\ell^{\iota}(y, z)\right|_{\rho}}\right| \leq \mathcal{O}\left(\left|\ell^{\iota}(y, w)\right|_{\rho}^{\alpha}\right),
$$

where $0<\alpha \leq 1$ and the constant of proportionality are uniform on $z \in \Lambda$. Therefore, the HR structure determined by $\phi$ is equal to the initial HR structure $\left(r^{s}, r^{u}\right)$, which ends the proof.

Now, let us construct the $C^{1+}$ structure $\mathcal{S}_{\mathrm{HR}}$. Let $\left\{M_{1}, \ldots, M_{n}\right\}$ be a Markov partition for $f$. For every Markov rectangle $M_{m}$, we take a rectangle $R_{m} \supset M_{m}$ which contains a small neighbourhood of $M_{m}$ with respect to the distance $d_{\Lambda}$. We construct an orthogonal chart $i_{m}: R_{m} \rightarrow \mathbb{R}^{2}$ as in Proposition 4.2. Let $h_{m, k}: i_{m}\left(R_{m} \cap R_{k}\right) \rightarrow i_{k}\left(R_{m} \cap R_{k}\right)$ be the map defined by $h_{m, k}(x)=i_{m} \circ i_{k}^{-1}(x)$. By Proposition 4.2, there is a $C^{1+}$ diffeomorphic extension $H_{m, k}: U_{m, k} \rightarrow U_{k, m}$ of $h_{m, k}$ which sends vertical lines into vertical lines and horizontal lines into horizontal lines. Let us denote by $S_{m}$ the rectangle in $\mathbb{R}^{2}$ whose boundary contains the image under $i_{m}$ of the boundary of $M_{m}$. For every pair of Markov rectangles $M_{m}$ and $M_{k}$ which intersect in a partial side $I_{m, k}=M_{m} \cap M_{k}$, let $J_{m, k}$ and $J_{k, m}$ be the smallest line segments containing respectively the sets $i_{m}\left(I_{m, k}\right)$ and $i_{k}\left(I_{m, k}\right)$. Hence, $J_{k, m}=H_{m, k}\left(J_{m, k}\right)$. Let $\tilde{M}=\bigsqcup_{m=1}^{n} S_{m} /\left\{H_{m, k}\right\}$ be the disjoint union of the squares $S_{m}$ where we identify two points $x \in J_{m, k}$ and $y \in J_{k, m}$ if $H_{k, m}(x)=y$. Hence, $\tilde{M}$ is a topological surface possibly with boundary. By taking appropriate extensions $E_{m}$ of the rectangles $S_{m}$ and using the maps $H_{m, k}$ to determine the identifications along the boundaries, we get a surface $\hat{M}=\bigsqcup_{m=1}^{n} E_{m} /\left\{H_{m, k}\right\}$ without boundary. The surface $\hat{M}$ has a natural smooth structure $\mathcal{S}_{\mathrm{HR}}$ that we now describe: if a point $z$ is contained in the interior of $E_{m}$ then we take a small open neighbourhood $U_{z}$ of $z$ contained in $E_{m}$ and we define a chart $u_{z}: U_{z} \rightarrow \mathbb{R}^{2}$ as being the inclusion of $U_{z} \cap E_{m}$ into $\mathbb{R}^{2}$. Otherwise $z$ is contained in a boundary of two or three or four sets $E_{m_{1}}, \ldots, E_{m_{n}}$ which we order such that the maps $I_{m_{1}, m_{2}}, \ldots, I_{m_{n}, m_{1}}$ are well-defined. In this case we take a small open neighbourhood $U_{z}$ of $z$ and we define the chart $u_{z}: U_{z} \rightarrow \mathbb{R}^{2}$ as follows: (i) $\quad u_{z} \mid\left(U_{z} \cap E_{n}\right)$ is the inclusion of $U_{z} \cap E_{n}$ into $\mathbb{R}^{2}$; and

(ii) $u_{z} \mid\left(U_{z} \cap E_{j}\right)=H_{m_{n-1}, m_{n}} \circ \cdots \circ H_{m_{j}, m_{j+1}}$ for $j \in\{1, \ldots, n-1\}$.

Since the maps $H_{m_{1}, m_{2}}, \ldots, H_{m_{n-1}, m_{n}}$ and $H_{m_{n}, m_{1}}$ are smooth, we obtain that the set of all these charts form a $C^{1+}$ structure $\mathcal{S}_{\mathrm{HR}}$ on $\hat{M}$.

We will also denote its embedding into $\hat{M}$ by $\Lambda$ and by a rectangle we also mean the embedding of a rectangle into $\hat{M}$. By Proposition 4.2 and by construction of the maps $H_{m, k}$, for every $z \in \Lambda$ and for every rectangle $R_{z}$ the orthogonal chart $i_{z}: R_{z} \rightarrow \mathbb{R}^{2}$ has an extension $v_{z}$ whose restriction to an open set $V_{z}$ of $z$ is a chart $C^{1+}$ compatible with the structure $\mathcal{S}_{\mathrm{HR}}$.

Proof of Theorem 5.3. Let $\phi$ be a $C^{r}$ hyperbolic realization of $(f, \Lambda, \mathcal{W})$. Let $c_{n}: I \rightarrow$ $[0,1]$ be defined as $d_{2, n} \circ d_{1, n}$, where $d_{1, n}: I \rightarrow f_{l}^{-n} I$ is given by $f_{l}^{-n}$, and $d_{2, n}=\lambda \circ i_{n}$, where $i_{n}: f_{l}^{-n} I \rightarrow \mathbb{R}$ is contained in a bounded $C^{r}$ lamination atlas with bounded geometry $\mathcal{A}_{\phi}^{\iota}$ induced by $\phi$ and $\lambda$ is the affine map of $\mathbb{R}$ which sends the endpoints of $i_{n}\left(f_{l}^{-n} I\right)$ into 0 and 1. Using (3.6), we obtain that $c=\lim c_{n}$ is a chart of the form given in (3.3) with respect to $r^{\iota}$ (up to scale) and $c$ is $C^{1+}$ compatible with the charts in $\mathcal{A}_{\phi}^{l}$. Since the atlas $\mathcal{A}_{\phi}^{l}$ has bounded geometry, the function $d_{2, n} \circ d_{1, n} \circ i_{0}^{-1}$ is the 
composition of an exponential contraction $i_{n} \circ d_{1, n} \circ i_{0}^{-1}$ in the $C^{r}$ norm followed by a linear map $\lambda$. Hence, there is $C>0$ such that the $C^{r}$ norm of $d_{2, n} \circ d_{1, n} \circ i_{0}^{-1}$ is bounded by $C$ for all $n \geq 0$. Thus, by Arzelá-Ascoli's Theorem we obtain that the sequence $d_{2, n} \circ d_{1, n} \circ i_{0}^{-1}$ converges in the $C^{r-\varepsilon}$ norm to a $C^{r}$ map $d$ with $C^{r}$ norm also bounded by $C$. Hence, $c=d \circ i_{0}^{-1}$ is $C^{r}$ compatible with the charts in $\mathcal{A}_{\phi}^{l}$ and so $\mathcal{A}\left(r^{l}\right)$ is a $C^{r}$ atlas. Thus, if the $\iota$-basic holonomies $\theta: I \rightarrow J$ are $C^{s}$ for some $1<s \leq r$ with respect to the charts in $\mathcal{A}_{\phi}^{l}$ then the basic holonomies are also $C^{s}$ with respect to the charts in $\mathcal{A}\left(r^{l}\right)$. Since by Proposition 3.7 the charts in $\mathcal{A}\left(r^{l}\right)$ do not depend upon the $C^{r^{\prime}}$ hyperbolic realizations $\psi$ which are $C^{1+}$ compatible with $\phi$, we obtain that the basic holonomies in $\mathcal{A}\left(r^{l}\right)$ attain at least the maximum smoothness of the basic holonomies with respect to any atlas $\mathcal{A}_{\psi}^{\iota}$ induced by these realizations $\psi$.

By construction of the structure $\mathcal{S}_{\mathrm{HR}}$ in Proposition 5.7, the smoothness of the hyperbolic representative in this structure and the smoothness of the basic holonomies in this structure are equal to the smoothness of the basic holonomies in $\mathcal{A}\left(r^{s}\right)$ and $\mathcal{A}\left(r^{u}\right)$, which ends the proof of part (i) of this theorem.

Let $\phi$ and $\psi$ be two $C^{r}$ Anosov diffeomorphisms which are $C^{1+}$ conjugated and let $\mathcal{A}_{\phi}^{\iota}$ and $\mathcal{A}_{\psi}^{\iota}$ be, respectively, $C^{r}$ atlases induced by $\phi$ and $\psi$ for $\iota \in\{s, u\}$. By Proposition 3.7, $\phi$ and $\psi$ determine the same pair of ratio functions $\left(r^{s}, r^{u}\right)$. As before the charts in $\mathcal{A}\left(r^{l}\right)$ are $C^{r}$ compatible with the charts in $\mathcal{A}_{\phi}^{l}$ and $\mathcal{A}_{\psi}^{l}$ and the overlap maps have $C^{r}$ uniformly bounded norm. Therefore, the conjugacy between $\phi$ and $\psi$ is $C^{r}$ along the stable and unstable leaves of the transverse stable and unstable foliations with uniformly smooth leaves. Hence, by Journé (see [7]) the conjugacy is $C^{r-\varepsilon}$ for all small $\varepsilon>0$, which ends the proof of part (ii) of this theorem.

\section{Solenoid functions}

We now discuss the solenoid functions that will provide us with our Teichmüller space. We show how to associate with each HR structure on $\Lambda$ a canonical pair of solenoid functions which will classify the HR structures.

For $\iota=s$ or $u$ let $\mathbf{S}^{\iota}$ denote the set of all ordered pairs $(I, J)$ where at least one of $I$ and $J$ is a primary cylinder, the other is either a primary cylinder or a primary gap and the intersection of $I$ and $J$ consists of a single endpoint (see $\$ 2.2$ for the definition of primary cylinders and gaps). Pairs $(I, J)$ where both are primary cylinders are called leaf-leaf pairs or otherwise leaf-gap pairs.

We define a pseudo-metric $d_{\mathbf{S}^{\iota}}: \mathbf{S}^{\iota} \times \mathbf{S}^{\iota} \rightarrow \mathbb{R}^{+}$on the set $\mathbf{S}^{\iota}$ by

$$
d_{\mathbf{S}^{\prime}}\left((I, J),\left(I^{\prime}, J^{\prime}\right)\right)=\max \left\{d_{\Lambda}\left(I, I^{\prime}\right), d_{\Lambda}\left(J, J^{\prime}\right)\right\} .
$$

Now suppose that we have an HR structure $\left(r^{s}, r^{u}\right)$ for $(f, \Lambda, \mathcal{W})$. Then for $\iota=s$ and $u$ this defines functions $s^{s}: \mathbf{S}^{s} \rightarrow \mathbb{R}^{+}$and $s^{u}: \mathbf{S}^{u} \rightarrow \mathbb{R}^{+}$by restricting the stable and unstable ratio functions $r^{s}$ and $r^{u}$ to the sets $\mathbf{S}^{s}$ and $\mathbf{S}^{u}$. We call such functions $s^{s}$ and $s^{u}$ realized solenoid functions and denote the set of such pairs of them by $\mathcal{S}(f, \Lambda, \mathcal{W})$. The main theorem of this section gives an abstract characterization of this set.

THEOREM 6.1. $\mathcal{S}(f, \Lambda, \mathcal{W})$ is the set of all function pairs $\left(s^{s}, s^{u}\right)$ where for $\iota=s$ and $u$, $s_{\iota}: \mathbf{S}^{\iota} \rightarrow \mathbb{R}^{+}$is Hölder continuous in $d_{\mathbf{S}^{\iota}}$ and satisfies $s_{\iota}(J, I)=s_{\iota}(I, J)^{-1}$ and both the $f$-matching and boundary conditions described later. 


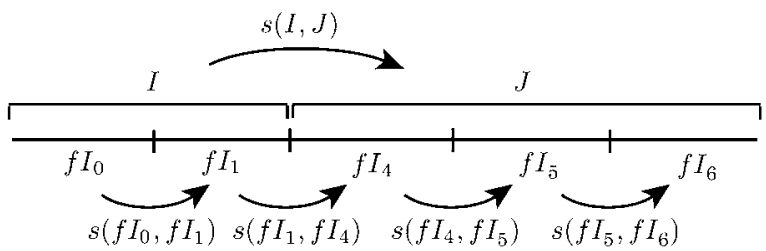

FIGURE 6. The $f$-matching condition.

In the discussion which follows we refer to Hölder continuous functions $s_{\iota}: \mathbf{S}^{\iota} \rightarrow \mathbb{R}^{+}$ satisfying $s_{\iota}(J, I)=s_{\iota}(I, J)^{-1}$ and the $f$-matching and boundary conditions as abstract solenoid functions. Of course our aim is to show that the abstract functions are the realized functions.

6.1. Hölder continuity of solenoid functions. This means that for $t=(I, J)$ and $t^{\prime}=\left(I^{\prime}, J^{\prime}\right)$ in $\mathbf{S}^{\iota},\left|s_{\iota}(t)-s_{\iota}\left(t^{\prime}\right)\right| \leq \mathcal{O}\left(\left(d_{\mathbf{S}^{\iota}}\left(t, t^{\prime}\right)\right)^{\alpha}\right)$. Clearly every realized solenoid function satisfies this by inequality (3.2).

6.2. The $f$-matching condition. This condition is a consequence of the $f$-invariance of the ratio functions. Consider an $\iota$-ratio function $r$ and let $(I, J) \in \mathbf{S}^{\iota}$. Suppose that we have pairs

$$
\left(I_{0}, I_{1}\right),\left(I_{1}, I_{2}\right), \ldots,\left(I_{n-2}, I_{n-1}\right) \in \mathbf{S}^{\iota}
$$

such that $I=\bigcup_{j=0}^{k-1} f_{\iota} I_{j}$ and $J=\bigcup_{j=k}^{n-1} f_{\iota} I_{j}$. Then it is easy to see that, by the $f$-invariance of $r$,

$$
r(I: J)=\frac{\sum_{j=0}^{k-1} \prod_{i=0}^{j-1} r\left(I_{i+1}: I_{i}\right)}{\sum_{j=k}^{n-1} \prod_{i=0}^{j-1} r\left(I_{i+1}: I_{i}\right)} .
$$

Since a realized solenoid function $s_{\iota}$ is the restriction of such an $r$ to such pairs it must satisfy the $f$-matching condition (see Figure 6 ). For all such pairs $(I, J)$ and $\left(I_{j}, J_{j}\right)$,

$$
s_{\iota}(I: J)=\frac{\sum_{j=0}^{k-1} \prod_{i=0}^{j-1} s_{\iota}\left(I_{i+1}: I_{i}\right)}{\sum_{j=k}^{n-1} \prod_{i=0}^{j-1} s_{\iota}\left(I_{i+1}: I_{i}\right)} .
$$

6.3. The boundary condition. Leaf segments $I$ in the boundaries of Markov rectangles can sometimes be written as the union of primary cylinders in more than one way. If $J$ is another leaf segment adjacent to $I$ then the value of $r(I: J)$ must be the same whichever decomposition we use. If we write $J=I_{0}=I_{0}^{\prime}$ and $I$ as $\bigcup_{i=1}^{s} I_{i}$ and $\bigcup_{j=1}^{s^{\prime}} I_{j}^{\prime}$ where the $I_{i}$ and $I_{j}^{\prime}$ are primary cylinders with $I_{i} \neq I_{j}^{\prime}$ for all $i$ and $j$, then the previous two ratios are

$$
\sum_{i=1}^{s} \prod_{j=1}^{i} r\left(I_{j}: I_{j-1}\right)=\sum_{i=1}^{s^{\prime}} \prod_{j=1}^{i} r\left(I_{j}^{\prime}: I_{j-1}^{\prime}\right) .
$$




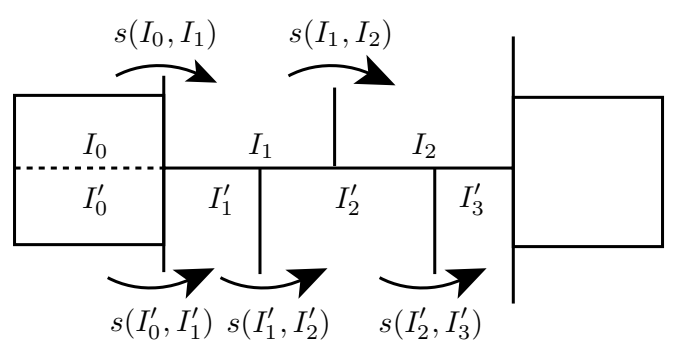

FIGURE 7. The boundary condition.

Thus a realized solenoid function $s_{\iota}$ must satisfy the boundary condition (see Figure 7) which is: for all such leaf-leaf pairs $\left(I_{j}, I_{j-1}\right) \in \mathbf{S}^{\iota}, j=1, \ldots, s$ and $\left(I_{j}^{\prime}, I_{j-1}^{\prime}\right) \in \mathbf{S}^{\iota}$, $j=1, \ldots, s^{\prime}$ with $I_{0}=I_{0}^{\prime}$ and with $\bigcup_{j=1}^{s} I_{j}=\bigcup_{j=1}^{s} I_{j}^{\prime}$ and $I_{i} \neq I_{j}^{\prime}$ for all $i$ and $j$

$$
\sum_{i=1}^{s} \prod_{j=1}^{i} s_{\iota}\left(I_{j}: I_{j-1}\right)=\sum_{i=1}^{s^{\prime}} \prod_{j=1}^{i} s_{\iota}\left(I_{j}^{\prime}: I_{j-1}^{\prime}\right)
$$

Proof of Theorem 6.1. Clearly every realized solenoid pair is an abstract one by this discussion.

Now we prove the converse. Since the solenoid functions are continuous and their domains are compact they are bounded away from 0 and $\infty$. By this boundedness and the $f$-matching condition of the solenoid functions and by iterating the domains $\mathbf{S}^{s}$ and $\mathbf{S}^{u}$ of the solenoid functions backward and forward by $f$, we determine the ratio functions $r^{s}$ and $r^{u}$ at very small (and large) scales, such that $f$ leaves the ratios invariant. Then, using the boundedness again, we extend the ratio functions to all pairs of small adjacent leaf segments by continuity.

By the boundary condition of the solenoid functions, the ratio functions are well determined at the boundaries of the Markov rectangles. The proof of Proposition 3.4 shows how to deduce inequality (3.4) from the Hölder continuity of the solenoid function.

Acknowledgements. A. A. Pinto was supported in part by the Gulbenkian Foundation, FCT, PRODYN-ESF, PRAXIS and Centro de Matemática Aplicada da Universidade do Porto.

D. A. Rand was supported in part by the Wolfson Foundation and the UK Science and Engineering Research Council.

We are grateful to Dennis Sullivan for a number of very fruitful discussions over a period of several years. In particular, he posed the question to us of how to modify his approach using scaling functions so as to parametrize $C^{1+}$ structures for expanding maps of the circle. With him we discovered how to link the resulting solenoid functions $[\mathbf{1 0 , 1 1}]$ with affine structures for smooth Markov maps (see [16]). The HR structures discussed here are a natural outgrowth of these ideas. 


\section{REFERENCES}

[1] R. Abraham and J. Robbin. Transversal Mappings and Flows. W. A. Benjamin, New York, 1967.

[2] E. Cawley. The Teichmüller space of an Anosov diffeomorphism of $T^{2}$. Inv. Math. 112 (1993), 351-376.

[3] J. Franks. Anosov diffeomorphisms on tori. Trans. Amer. Math. Soc. 145 (1969), 117-124.

[4] R. Llave, J. M. Marco and R. Moriyon. Canonical perturbation theory of Anosov systems and regularity results for the Livsic cohomology equations. Ann. Math. 123 (1986), 537-612.

[5] R. Llave. Invariants for smooth conjugacy of hyperbolic systems. Preprint.

[6] M. Hirsch and C. Pugh. Stable manifolds and hyperbolic sets. Proceedings of Symposia in Pure Mathematics, Vol. 14. American Mathematical Society, Providence, RI, 1970, pp. 133-164.

[7] J. L. Journé. A regularity lemma for functions of several variables. Rev. Math. Iberoamer. 4 (1988), 187193.

[8] R. Mañé. Ergodic Theory and Differentiable Dynamics. Springer, Berlin, 1987.

[9] S. Newhouse. On codimension one Anosov diffeomorphisms. Amer. J. Math. 92 (1970), 671-762.

[10] A. A. Pinto and D. A. Rand. Classifying $C^{1+}$ structures on hyperbolical fractals: 1 . The moduli space of solenoid functions for Markov maps on train-tracks. Ergod. Th. \& Dynam. Sys. 15 (1995), 697-734.

[11] A. A. Pinto and D. A. Rand. Classifying $C^{1+}$ structures on hyperbolical fractals: 2. Embedded trees. Ergod. Th. \& Dynam. Sys. 15 (1995), 969-992.

[12] A. A. Pinto and D. A. Rand. Existence, uniqueness and ratio decomposition for Gibbs states via duality. Ergod. Th. \& Dynam. Sys. 21 (2001), 533-543.

[13] A. A. Pinto and D. A. Rand. Geometric measures for hyperbolic surface dynamics. In preparation.

[14] A. A. Pinto and D. A. Rand. Smoothness of holonomies for codimension 1 hyperbolic dynamics. Bull. London Math. Soc. 34 (2002), 341-352.

[15] A. A. Pinto and D. A. Rand. Rigidity of hyperbolic surface dynamics. Submitted.

[16] A. A. Pinto and D. Sullivan. Asymptotic geometry applied to dynamical systems. Submitted.

[17] M. Shub. Global Stability of Dynamical Systems. Springer, Berlin, 1987.

[18] D. Sullivan. Differentiable structures on fractal-like sets determined by intrinsic scaling functions on dual Cantor sets. Proceedings of Symposia in Pure Mathematics, Vol. 48. American Mathematical Society, Providence, RI, 1988.

[19] D. Sullivan. Bounds, quadratic differentials, and renormalization conjectures. Amer. Math. Soc. Centennial Publications. Volume 2: Mathematics into the Twenty-first Century (1988 Centennial Symposium, 8-12 August). American Mathematical Society, Providence, RI, 1991. 